\title{
Stochastic dominance with imprecise information
}

\author{
Ignacio Montes*, Enrique Miranda, Susana Montes \\ University of Oviedo, Dep. of Statistics and Operations Research.
}

\begin{abstract}
Stochastic dominance, which is based on the comparison of distribution functions, is one of the most popular preference measures. However, its use is limited to the case where the goal is to compare pairs of distribution functions, whereas in many cases it is interesting to compare sets of distribution functions: this may be the case for instance when the available information does not allow to fully elicitate the probability distributions of the random variables. To deal with these situations, a number of generalisations of the notion of stochastic dominance are proposed; their connection with an equivalent p-box representation of the sets of distribution functions is studied; a number of particular cases, such as sets of distributions associated to possibility measures, are investigated; and an application to the comparison of the Lorenz curves of countries within the same region is presented.
\end{abstract}

Keywords: Distribution function, stochastic dominance, probability boxes, possibility measures, Lorenz curves.

\section{Introduction}

The comparison of random variables is a natural problem that arises in many fields, and for this reason there are many different proposals in stochastic ordering. One of the most popular is stochastic dominance. First degree stochastic dominance considers one random variable greater than another one when the first one is more likely to take greater values. Although this notion has been employed since the 1930s, it has been in the last decades when it has witnessed increasing popularity; this is testified by the applications of stochastic dominance many different areas, such as economics [7], social welfare [4], agriculture [26], operational research [37], etc.

It is not uncommon, however, to encounter situations where there is uncertainty about the probability distributions underlying the random variables of interest; we may for instance have vague or conflicting information, or errors in the transmission of the available data. This results in the impossibility of eliciting the probability distribution with certain guarantees. Instead, we may consider more realistic to work with sets of probability distributions which are sure to include the 'true' one. In other situations, we may be able to work with precise probability measures, but we may be interested in comparing the sets of

\footnotetext{
${ }^{*}$ Corresponding author. Tel:(+34) 985186466, Fax: $(+34) 985103354$.

Email addresses: imontes@uniovi.es (Ignacio Montes), mirandaenrique@uniovi.es (Enrique Miranda), montes@uniovi.es (Susana Montes)
} 
probability distributions induced by random variables that share some common features.

Our goal in this paper is to extend the notion of stochastic dominance to the comparison of sets of distribution functions, following the steps made by Denoeux for the particular case of belief and plausibility measures [13]. After giving some preliminary concepts in Section 2, we overview in Section 3 the work by Denoeux and generalise his work to arbitrary sets of probability measures. We investigate the relationships between the different definitions and study their main properties, detailing their connection with the notions proposed in [13].

In Section 4, we investigate a number of particular cases which are of interest in relation to other works, such as the comparison of possibility measures by means of stochastic dominance or the comparison of two sets of distribution functions with the same lower and upper bounds. Our work is illustrated in Section 5 with an application to the comparison of sets of Lorenz curves. Finally, in Section 6 we discuss the main implications of our results and future lines of research. We have gathered a number of examples in an appendix.

We shall restrict our work to random variables taking values on the unit interval; since this is homeomorphic to any closed interval on the real line, the results extend immediately to distribution functions taking values on any interval $[a, b]$, where $a<b \in \mathbb{R}$. In fact, our work can be easily generalised to any totally ordered space, simply by adding a smallest and greatest value. On the other hand, we shall work with sets of $\sigma$-additive probability measures, to be closer to the usual works on stochastic dominance. This gives rise, however, to a number of additional complications with respect to the usual works with non-additive measures, which are based on sets of finitely additive probability measures. To which extent this assumption makes a difference in the corresponding results shall be discussed in Section 3 .

\section{Preliminary concepts}

\subsection{Stochastic dominance}

The notion of stochastic dominance between random variables is based on the comparison of their corresponding distribution functions. In this paper, we are going to work with random variables taking values on $[0,1]$. The distribution function is thus defined in the following way:

Definition 1. A cumulative distribution function (cdf) is a function $F:[0,1] \rightarrow$ $[0,1]$ satisfying the following properties:

- $x \leq y \Rightarrow F(x) \leq F(y) \forall x, y$ [Monotonicity].

- $F(1)=1$ [Normalisation].

- $F(x)=\lim _{\epsilon \downarrow 0} F(x+\epsilon) \forall x<1$ [Right-continuity].

When $F$ satisfies the properties of monotonicity and normalisation, it is associated to a finite additive probability distribution, and we shall call it a finitely additive distribution function.

One of the most popular methods for the comparison of cdf is stochastic dominance $[30,36,44]$ : 
Definition 2. Given two cumulative distribution functions $F$ and $G$, we say that $F$ stochastically dominates $G$, and denote it $F \succeq_{\mathrm{FSD}} G$, if

$$
F(t) \leq G(t) \text { for every } t \in[0,1] .
$$

This definition produces a partial order in the space of cumulative distribution functions, from which we can derive the notions of strict stochastic dominance, indifference and incomparability:

- We say that $F$ strictly stochastically dominates $G$, and denote it by $F \succ_{\text {FSD }} G$, if $F \succeq_{\text {FSD }} G$ but $G \succeq_{\text {FSD }} F$. This holds if and only if $F \leq G$ and there is some $t \in[0,1]$ such that $F(t)<G(t)$.

- $F$ and $G$ are stochastically indifferent if $F \succeq_{\mathrm{FSD}} G$ and $G \succeq_{\mathrm{FSD}} F$, or equivalently, if $F=G$.

- $F$ and $G$ are stochastically incomparable if $F \nsucceq_{\mathrm{FSD}} G$ and $G \nsucceq_{\mathrm{FSD}} F$.

Stochastic dominance is commonly used in economics and finance [14, 24] and can be given the following interpretation: $F \succeq_{\text {FSD }} G$ means that the choice of $F$ over $G$ is rational, in the sense that we prefer the alternative that provides greater probability of having a greater profit. The notion has also been used in other frameworks such as reliability theory, statistical physics, epidemiology, etc (see $[30,36,44]$ for more information).

\subsection{P-boxes}

Our goal in this paper is to extend the notion of stochastic dominance to the case where we consider sets of probability measures instead of a single one. As a consequence, we shall work within the theory of imprecise probabilities. The term imprecise probability [48] refers to uncertainty models applicable in situations where the available information does not allow us to single out a unique probability measure for all random variables involved. Examples of such models include 2- and $n$-monotone capacities [11], lower and upper previsions [48], belief functions [1, 43], credal sets [29], possibility and necessity measures [16], interval probabilities [49], and coherent risk measures [3].

One such model is considered in this paper: pairs of lower and upper distribution functions, also called probability boxes, or briefly, p-boxes [18, 19]. P-boxes are often used in risk or safety studies, in which cumulative distributions play an essential role. Many theoretical properties and practical aspects of p-boxes have already been studied in the literature. They have been connected to other uncertainty models, such as random sets [28] and possibility measures [47], and have been applied in different contexts [21,38].

Definition 3. A probability box, or p-box for short, $(\underline{F}, \bar{F})$ is the set of cumulative distribution functions bounded between two finitely additive distribution functions $\underline{F} \leq \bar{F}$. We shall refer to $\underline{F}$ as the lower distribution function and to $\bar{F}$ as the upper distribution function of the $p$-box.

Note that $\underline{F}, \bar{F}$ need not be cumulative distribution functions, and as such they need not belong to the set $(\underline{F}, \bar{F})$; they are only required to be finitely additive distribution functions. 
If we let $\mathcal{F}$ be a set of cumulative distribution functions, its associated $p$-box $(\underline{F}, \bar{F})$ is determined by

$$
\underline{F}(x):=\inf _{F \in \mathcal{F}} F(x), \bar{F}(x):=\sup _{F \in \mathcal{F}} F(x) \forall x \in[0,1] .
$$

We shall determine later to which extent the preferences between two sets of cumulative distribution functions coincide with the preferences between their associated p-boxes, and if furthermore these preferences can be summarised by the lower and upper distribution functions they induce.

Note that $\mathcal{F}$ need not coincide with the set of distribution functions associated to the p-box $(\underline{F}, \bar{F})$ (it may be a proper subset) and on the other hand $\underline{F}$ and $\bar{F}$ need not belong to $(\underline{F}, \bar{F})$ : although the infimum $\underline{F}$ of a set of cumulative distribution functions is again a cumulative distribution function, their supremum may be only a finitely additive distribution function (i.e., it need not be right-continuous), and as a consequence it need not belong to the $p$-box $(\underline{F}, \bar{F})$. That p-boxes are usually less informative than sets of probability measures, and may thus increase the imprecision, is already well-known (see for instance $[5,15,28]$ for comments on this in the context of random set theory).

\section{Imprecise stochastic dominance}

A first approach to the extension of stochastic dominance to an imprecise framework was made by Denoeux in [13].

\subsection{Stochastic dominance for belief and plausibility measures}

Consider two random variables $U$ and $V$ such that $P(U \leq V)=1$. They can be equivalently represented as a random interval $[U, V]$, which in turn [12] induces the following belief and plausibility functions:

$$
\operatorname{bel}(A)=P([U, V] \subseteq A) \text { and } p l(A)=P([U, V] \cap A \neq \emptyset)
$$

for every element $A$ in the Borel sigma-algebra $\mathfrak{B}(\mathbb{R})$. This implies that

$$
\operatorname{bel}((-\infty, x])=F_{V}(x) \text { and } p l((-\infty, x])=F_{U}(x) \forall x \in \mathbb{R} .
$$

The associated set of probability measures $\mathcal{P}$ is given by:

$$
\mathcal{P}=\{P \text { probability }: \operatorname{bel}(A) \leq P(A) \leq p l(A) \text { for every } A \in \mathfrak{B}(\mathbb{R})\} .
$$

Now, if we consider two random closed intervals $[U, V]$ and $\left[U^{\prime}, V^{\prime}\right]$, one possible way of comparing them is to compare their associated sets of probabilities:

$$
\begin{aligned}
& \mathcal{P}=\{P \text { probability }: \operatorname{bel}(A) \leq P(A) \leq p l(A) \text { for every } A \in \mathfrak{B}(\mathbb{R})\} \\
& \mathcal{P}^{\prime}=\left\{P \text { probability }: \operatorname{bel}^{\prime}(A) \leq P(A) \leq p l^{\prime}(A) \text { for every } A \in \mathfrak{B}(\mathbb{R})\right\}
\end{aligned}
$$

where $b e l^{\prime}$ and $p l^{\prime}$ are the belief and plausibility functions induced by $\left[U^{\prime}, V^{\prime}\right]$, respectively.

Based on the usual ordering between real intervals (see [20]), Denoeux [13] proposed the following notions:

- $\mathcal{P} \gg \mathcal{P}^{\prime} \Leftrightarrow p l^{\prime}((x, \infty)) \leq \operatorname{bel}((x, \infty))$ for every $x \in \mathbb{R}$. 
- $\mathcal{P}>\mathcal{P}^{\prime} \Leftrightarrow p l^{\prime}((x, \infty)) \leq p l((x, \infty))$ for every $x \in \mathbb{R}$.

- $\mathcal{P} \geqslant \mathcal{P}^{\prime} \Leftrightarrow \operatorname{bel}^{\prime}((x, \infty)) \leq \operatorname{bel}((x, \infty))$ for every $x \in \mathbb{R}$.

- $\mathcal{P} \gtrsim \mathcal{P}^{\prime} \Leftrightarrow b e l^{\prime}((x, \infty)) \leq p l((x, \infty))$ for every $x \in \mathbb{R}$.

The above notions can be characterised in terms of the stochastic dominance between the lower and upper limits of the random intervals:

Proposition 1. [13, Proposition 3] Let $[U, V]$ and $\left[U^{\prime}, V^{\prime}\right]$ be two random closed intervals, and let $\mathcal{P}$ and $\mathcal{P}^{\prime}$ their associated sets of probability measures. The following equivalences hold:

- $\mathcal{P} \gg \mathcal{P}^{\prime} \Leftrightarrow U \succeq_{\mathrm{FSD}} V^{\prime}$.

- $\mathcal{P}>\mathcal{P}^{\prime} \Leftrightarrow U \succeq_{\mathrm{FSD}} U^{\prime}$.

- $\mathcal{P} \geqslant \mathcal{P}^{\prime} \Leftrightarrow V \succeq_{\mathrm{FSD}} V^{\prime}$.

- $\mathcal{P} \gtrsim \mathcal{P}^{\prime} \Leftrightarrow V \succeq_{\mathrm{FSD}} U^{\prime}$.

Moreover, they can also be represented in an equivalent way by means of p-boxes: if we consider the set of distribution functions induced by $\mathcal{P}$, we obtain

$$
\left\{F: F_{V} \leq F \leq F_{U}\right\},
$$

i.e., the p-box determined by $F_{V}$ and $F_{U}$. Similarly, the set $\mathcal{P}^{\prime}$ induces the p-box $\left(F_{V^{\prime}}, F_{U^{\prime}}\right)$, and Denoeux's definitions are equivalent to comparing the lower and upper distribution functions of these p-boxes, as we can see from Proposition 1. Note moreover that the same result holds if we consider finitely additive probability measures instead of $\sigma$-additive ones, because both of them determine the same $p$-box and the lower and upper distribution functions are included in both cases.

\subsection{Extension to pairs of sets of distribution functions}

One important property of the sets $\mathcal{P}, \mathcal{P}^{\prime}$ considered above is that the sets of distribution functions they induce include their lower and upper distributions. As we shall see, this is not necessarily the case when we start from arbitrary sets of probability measures, and as a consequence their comparison by means of the lower and upper distribution functions may produce a loss of information.

In this section, we propose a number of definitions that extend Denoeux' ones to arbitrary sets of probability measures. Given a set of probability measures $\mathcal{P}$ on $[0,1]$, we shall denote by $\mathcal{F}=\left\{F_{P}: P \in \mathcal{P}\right\}$ its associated set of cumulative distribution functions.

Definition 4 . Let $\mathcal{P}_{1}, \mathcal{P}_{2}$ be two sets of probability measures on $[0,1]$, and let $\mathcal{F}_{1}, \mathcal{F}_{2}$ be their associated sets of distribution functions. We say that $\mathcal{P}_{1}$ :

- $\left(F S D_{1}\right)$ stochastically dominates $\mathcal{P}_{2}$, and denote it by $\mathcal{F}_{1} \succeq_{\mathrm{FSD}_{1}} \mathcal{F}_{2}$ if and only if for every $F_{1} \in \mathcal{F}_{1}, F_{2} \in \mathcal{F}_{2}$ it holds that $F_{1} \leq F_{2}$.

- $\left(F S D_{2}\right)$ stochastically dominates $\mathcal{P}_{2}$, and denote it by $\mathcal{F}_{1} \succeq_{\mathrm{FSD}_{2}} \mathcal{F}_{2}$ if and only if there is some $F_{1} \in \mathcal{F}_{1}$ such that $F_{1} \leq F_{2}$ for every $F_{2} \in \mathcal{F}_{2}$.

- $\left(F S D_{3}\right)$ stochastically dominates $\mathcal{P}_{2}$ and denote it by $\mathcal{F}_{1} \succeq_{\mathrm{FSD}_{3}} \mathcal{F}_{2}$ if and only if for every $F_{2} \in \mathcal{F}_{2}$ there is some $F_{1} \in \mathcal{F}_{1}$ such that $F_{1} \leq F_{2}$. 
- $\left(F S D_{4}\right)$ stochastically dominates $\mathcal{P}_{2}$, and denote it by $\mathcal{F}_{1} \succeq_{\mathrm{FSD}_{4}} \mathcal{F}_{2}$ if and only if there are $F_{1} \in \mathcal{F}_{1}, F_{2} \in \mathcal{F}_{2}$ such that $F_{1} \leq F_{2}$.

- $\left(F S D_{5}\right)$ stochastically dominates $\mathcal{P}_{2}$, and denote it by $\mathcal{F}_{1} \succeq_{\mathrm{FSD}_{5}} \mathcal{F}_{2}$ if and only if there is $F_{2} \in \mathcal{F}_{2}$ such that $F_{1} \leq F_{2}$ for every $F_{1} \in \mathcal{F}_{1}$.

- $\left(F S D_{6}\right)$ stochastically dominates $\mathcal{P}_{2}$, and denote it by $\mathcal{F}_{1} \succeq_{\mathrm{FSD}_{6}} \mathcal{F}_{2}$ if and only if for every $F_{1} \in \mathcal{F}_{1}$ there is $F_{2} \in \mathcal{F}_{2}$ such that $F_{1} \leq F_{2}$.
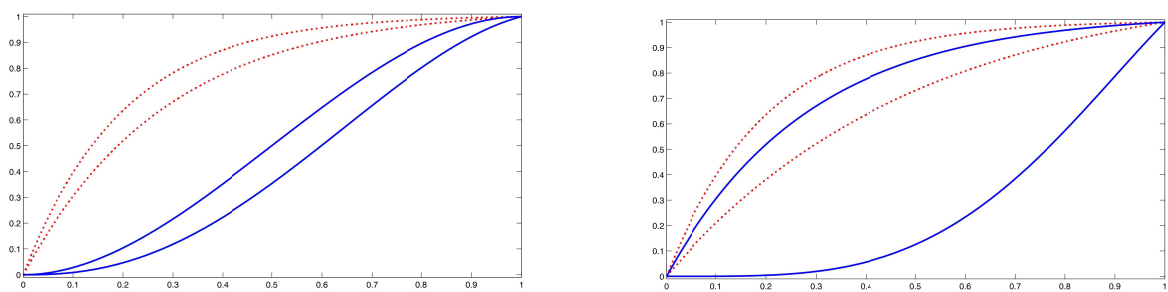

Figure 1: Examples of the several definitions of imprecise stochastic dominance.

An illustration of the above definitions is given in Figure 1, where we compare the set of distribution functions represented by a continuous line (that we shall call continuous distributions in this paragraph) with the set of distribution functions represented by a dotted line (that we shall call dotted distributions).

On the one hand, in the left picture the set of continuous distributions $\left(F S D_{1}\right)$-stochastically dominates the set of dotted distributions. In the right picture, there is a continuous distribution that dominates all dotted distributions, and a dotted distribution which is dominated by all continuous distributions. This means that the set of continuous distributions stochastically dominates the set of dotted distributions with respect to second to sixth definitions. Since there is also a dotted distribution that is dominated by a continuous distribution, we deduce that the set of continuous distributions and the set of dotted distributions are equivalent with respect to the fourth definition.

The conditions in Definition 4 can be given the following interpretation. $\left(F S D_{1}\right)$ means that any distribution function in $\mathcal{F}_{1}$ stochastically dominates any distribution function in $\mathcal{F}_{2}$, and as such it is related to the idea of interval dominance from decision making with sets of probabilities [50]. Conditions $\left(F S D_{2}\right)$ and $\left(F S D_{3}\right)$ mean that the 'best' case in $\mathcal{F}_{1}$ is better than the 'best' case in $\mathcal{F}_{2}$. The difference between them lies in whether there is a maximal element in $\mathcal{F}_{1} \cup \mathcal{F}_{2}$ in the partial order determined by stochastic dominance. These two conditions are related to the $\Gamma$-maximax criteria considered in [42]. On the other hand, conditions $\left(F S D_{5}\right)$ and $\left(F S D_{6}\right)$ mean that the 'worst' case in $\mathcal{F}_{1}$ is better than the 'worst' one in $\mathcal{F}_{2}$, and are related to the $\Gamma$-maximin criteria in $[8,22]$. Again, the difference between them lies in whether there is a minimum element in the order determined by stochastic dominance or not. Finally, $\left(F S D_{4}\right)$ is a weakened version of $\left(F S D_{1}\right)$, in the sense that it only requires that some element in $\mathcal{F}_{1}$ is preferred to some other element in $\mathcal{F}_{2}$, instead of requiring it for any pair in $\mathcal{F}_{1}, \mathcal{F}_{2}$. 
Remark 1. None of the above definitions induces an order, in the sense that none of the relationships $\succeq_{\mathrm{FSD}_{\mathrm{i}}}$ we can define on the sets of distribution functions satisfies simultaneously the properties of reflexivity, antisymmetry and transitivity. Specifically, it is not difficult to show that:

- $\succeq_{\mathrm{FSD}_{1}}$ is transitive and antisymmetric, but not reflexive.

- $\succeq_{\mathrm{FSD}_{2}}$ and $\succeq_{\mathrm{FSD}_{5}}$ are transitive, but they are neither reflexive nor antisymmetric.

- $\succeq_{\mathrm{FSD}_{3}}$ and $\succeq_{\mathrm{FSD}_{6}}$ are transitive and reflexive but they are not antisymmetric.

- $\succeq_{\mathrm{FSD}_{4}}$ is reflexive, but it is neither transitive nor antisymmetric.

Moreover, even if $\left(F S D_{1}\right)$ induces the only antisymmetric relation, it can never be applied in the imprecise case: $\mathcal{F}_{1} \succeq_{\mathrm{FSD}_{1}} \mathcal{F}_{2}$ and $\mathcal{F}_{2} \succeq_{\mathrm{FSD}_{1}} \mathcal{F}_{1}$ imply that $\mathcal{F}_{1}=\mathcal{F}_{2}=\{F\}$.

From any of these definitions we can infer immediately a relation of strict stochastic dominance and another one of stochastic indifference: we have

$$
\mathcal{F}_{1} \succ_{\mathrm{FSD}_{\mathrm{i}}} \mathcal{F}_{2} \Leftrightarrow \mathcal{F}_{1} \succeq_{\mathrm{FSD}_{\mathrm{i}}} \mathcal{F}_{2} \text { and } \mathcal{F}_{2} \succeq_{\mathrm{FSD}_{\mathrm{i}}} \mathcal{F}_{1}
$$

and

$$
\mathcal{F}_{1} \equiv_{\mathrm{FSD}_{\mathrm{i}}} \mathcal{F}_{2} \Leftrightarrow \mathcal{F}_{1} \succeq_{\mathrm{FSD}_{\mathrm{i}}} \mathcal{F}_{2} \text { and } \mathcal{F}_{2} \succeq_{\mathrm{FSD}_{\mathrm{i}}} \mathcal{F}_{1},
$$

and this for any $i=1, \ldots, 6$. Moreover, we shall say that $\mathcal{F}_{1}$ and $\mathcal{F}_{2}$ are incomparable with respect to $\left(F S D_{i}\right)$ when $\mathcal{F}_{1} \Varangle_{\mathrm{FSD}_{\mathrm{i}}} \mathcal{F}_{2}$ and $\mathcal{F}_{2} \Varangle_{\mathrm{FSD}_{\mathrm{i}}} \mathcal{F}_{1}$.

The relationships between these definitions are depicted in the following proposition.

Proposition 2. Let $\mathcal{F}_{1}, \mathcal{F}_{2}$ be two sets of cdfs on $[0,1]$.

a) The implications between the conditions of stochastic dominance are given by Figure 2.

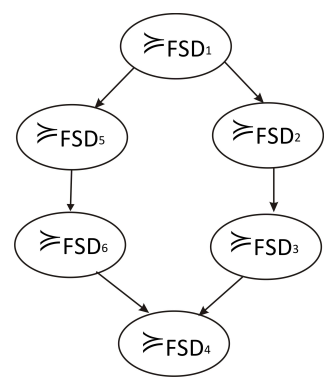

Figure 2: Relationships between the different definitions.

b) Concerning strict stochastic dominance,

- $\left(F S D_{2}\right)$ strict stochastic dominance implies $\left(F S D_{3}\right)$ strict stochastic dominance. 
- $\left(F S D_{5}\right)$ strict stochastic dominance implies $\left(F S D_{6}\right)$ strict stochastic dominance.

Proof. The first part follows immediately from the definitions, so we only prove the statement about strict stochastic dominance. To see that $\succ_{\mathrm{FSD}_{2}} \Rightarrow \succ_{\mathrm{FSD}_{3}}$, note that from the first part, if $\mathcal{F}_{1} \succ_{\mathrm{FSD}_{2}} \mathcal{F}_{2}$ then $\mathcal{F}_{1} \succeq_{\mathrm{FSD}_{3}} \mathcal{F}_{2}$. To see that there is strict preference, assume that $\mathcal{F}_{1} \equiv_{\mathrm{FSD}_{3}} \mathcal{F}_{2}$. Then given the $F_{1} \in \mathcal{F}_{1}$ such that $F_{1} \leq F_{2} \forall F_{2} \in \mathcal{F}_{2}$, there should be some $F_{2}^{\prime} \in \mathcal{F}_{2}$ such that $F_{2}^{\prime} \leq F_{1}$, whence $F_{1}=F_{2}^{\prime}$ and therefore $\mathcal{F}_{1} \equiv_{\mathrm{FSD}_{2}} \mathcal{F}_{2}$, a contradiction.

The proof of the implication $\succ_{\mathrm{FSD}_{5}} \Rightarrow \succ_{\mathrm{FSD}_{6}}$ is similar.

Example 1 in the Appendix shows that there is no additional implication between the notions of stochastic dominance or strict stochastic dominance. It is not difficult to see that the same implications represented in Figure 2 also hold for the derived relationships of stochastic indifference, and that there is no additional implication.

Remark 2. In some cases, it may be interesting to combine some of these definitions, for instance to consider $\mathcal{F}_{1}$ preferred to $\mathcal{F}_{2}$ when it is preferred according to definitions $\left(F S D_{2}\right)$ and $\left(F S D_{5}\right)$. Taking into account the implications depicted in Proposition 2, the combinations that produce new conditions are those where we take one condition out of $\left\{F S D_{2}, F S D_{3}\right\}$ together with one out of $\left\{F S D_{5}, F S D_{6}\right\}$.

If we combine for instance $\left(F S D_{2}\right)$ with $\left(F S D_{5}\right)$, so that

$$
\mathcal{F}_{1} \succeq \mathcal{F}_{2} \Leftrightarrow \mathcal{F}_{1} \succeq_{\mathrm{FSD}_{2}} \mathcal{F}_{2} \text { and } \mathcal{F}_{1} \succeq_{\mathrm{FSD}_{5}} \mathcal{F}_{2}
$$

we would be requiring that $\mathcal{F}_{1}$ has a best-case scenario which is better than any situation in $\mathcal{F}_{2}$ and that $\mathcal{F}_{2}$ has a worst-case scenario which is worse than any situation in $\mathcal{F}_{1}$. This turns out to be an intermediate condition between $\left(F S D_{1}\right)$ and each of $\left(F S D_{2}\right),\left(F S D_{5}\right)$, and it is not difficult to show that it is not equivalent to any of them.

Related results can be found in [13, Proposition 2] and in Section 4.1 later on; however, note that in both cases we consider we consider a situation of increase of the imprecision, which, in the context of [13], ends up producing the relationships $\mathcal{F}_{1} \succeq_{\mathrm{FSD}_{2}} \mathcal{F}_{2}$ and $\mathcal{F}_{2} \succeq_{\mathrm{FSD}_{5}} \mathcal{F}_{1}$.

Stochastic dominance between sets of probabilities can be studied by means of a p-box representation. Given any set of probability measures $\mathcal{P}$, its associated set of distribution functions $\mathcal{F}$ induces a $p$-box $(\underline{F}, \bar{F})$ by means of Eq. (2). Our next result relates the different definitions of stochastic dominance for sets of probabilities to their associated p-box representation:

Proposition 3. Let $\mathcal{F}_{1}, \mathcal{F}_{2}$ be two sets of cumulative distribution functions, and denote by $\left(\underline{F}_{1}, \bar{F}_{1}\right)$ and $\left(\underline{F}_{2}, \bar{F}_{2}\right)$ the p-boxes they induce.

1. $\mathcal{F}_{1} \succeq_{\mathrm{FSD}_{1}} \mathcal{F}_{2} \Leftrightarrow \bar{F}_{1} \leq \underline{F}_{2}$.

2. $\mathcal{F}_{1} \succeq_{\mathrm{FSD}_{2}} \mathcal{F}_{2} \Rightarrow \underline{F}_{1} \leq \underline{F}_{2}$.

3. $\mathcal{F}_{1} \succeq_{\mathrm{FSD}_{3}} \mathcal{F}_{2} \Rightarrow \underline{F}_{1} \leq \underline{F}_{2}$.

4. $\mathcal{F}_{1} \succeq_{\mathrm{FSD}_{4}} \mathcal{F}_{2} \Rightarrow \underline{F}_{1} \leq \bar{F}_{2}$.

5. $\mathcal{F}_{1} \succeq_{\mathrm{FSD}_{5}} \mathcal{F}_{2} \Rightarrow \bar{F}_{1} \leq \bar{F}_{2}$.

6. $\mathcal{F}_{1} \succeq_{\mathrm{FSD}_{6}} \mathcal{F}_{2} \Rightarrow \bar{F}_{1} \leq \bar{F}_{2}$. 
Proof. (1) Note that $\mathcal{F}_{1} \succeq_{\mathrm{FSD}_{1}} \mathcal{F}_{2}$ if and only if $F_{1} \leq F_{2}$ for every $F_{1} \in$ $\mathcal{F}_{1}, F_{2} \in \mathcal{F}_{2}$, and this is equivalent to $\bar{F}_{1}=\sup _{F_{1} \in \mathcal{F}_{1}} F_{1} \leq \inf _{F_{2} \in \mathcal{F}_{2}} F_{2}=\underline{F}_{2}$.

(3) By hypothesis, for every $F_{2} \in \mathcal{F}_{2}$ there is some $F_{1} \in \mathcal{F}_{1}$ such that $F_{1} \leq F_{2}$. As a consequence, $\underline{F}_{1} \leq F_{2} \forall F_{2} \in \mathcal{F}_{2} \Rightarrow \underline{F}_{1} \leq \inf _{F_{2} \in \mathcal{F}_{2}} F_{2}=\underline{F}_{2}$.

(4) If there are $F_{1} \in \mathcal{F}_{1}$ and $F_{2} \in \mathcal{F}_{2}$ s.t. $F_{1} \leq F_{2}$, then $\underline{F}_{1} \leq F_{1} \leq F_{2} \leq \bar{F}_{2}$.

(6) If for every $F_{1} \in \mathcal{F}_{1}$ there is some $F_{2} \in \mathcal{F}_{2}$ such that $F_{1} \leq F_{2}$, then $\bar{F}_{1}=\sup _{F_{1} \in \mathcal{F}_{1}} F_{1} \leq \sup _{F_{2} \in \mathcal{F}_{2}} F_{2}=\bar{F}_{2}$.

$(2,5)$ The second (resp. fifth) statement follows from the third (resp., sixth) and Proposition 2.

To see that the converse implications in the second to sixth statements do not hold in general, we refer to Example 2 in the Appendix.

As we mentioned after Definition 4 , the difference between $\left(F S D_{2}\right)$ and $\left(F S D_{3}\right)$ lies on whether the set of distribution functions $\mathcal{F}_{1}$ has a 'best case', i.e., a smallest distribution function; similarly, the difference between $\left(F S D_{5}\right)$ and $\left(F S D_{6}\right)$ lies on whether $\mathcal{F}_{2}$ has a greatest distribution function. Taking this into account, we can easily prove the equivalence between the above conditions when the lower or the upper distribution functions of the associated $p$-box belong to the set:

Proposition 4. Let us consider two sets of cumulative distribution functions $\mathcal{F}_{1}$ and $\mathcal{F}_{2}$

1. $\underline{F}_{1} \in \mathcal{F}_{1} \Rightarrow\left[\mathcal{F}_{1} \succeq_{\mathrm{FSD}_{2}} \mathcal{F}_{2} \Leftrightarrow \mathcal{F}_{1} \succeq_{\mathrm{FSD}_{3}} \mathcal{F}_{2}\right]$.

2. $\bar{F}_{2} \in \mathcal{F}_{2} \Rightarrow\left[\mathcal{F}_{1} \succeq_{\mathrm{FSD}_{5}} \mathcal{F}_{2} \Leftrightarrow \mathcal{F}_{1} \succeq_{\mathrm{FSD}_{6}} \mathcal{F}_{2}\right]$.

Proof. To see the first statement, use that by Proposition $2 \mathcal{F}_{1} \succeq_{\mathrm{FSD}_{2}} \mathcal{F}_{2} \Rightarrow$ $\mathcal{F}_{1} \succeq_{\mathrm{FSD}_{3}} \mathcal{F}_{2}$. Moreover, $\mathcal{F}_{1} \succeq_{\mathrm{FSD}_{3}} \mathcal{F}_{2}$ if and only if for every $F_{2} \in \mathcal{F}_{2}$ there is $F_{1} \in \mathcal{F}_{1}$ such that $F_{1} \leq F_{2}$. In particular, since $\underline{F}_{1} \leq F_{1}$ for every $F_{1} \in \mathcal{F}_{1}$, it holds that $\underline{F}_{1} \leq F_{2}$ for every $F_{2} \in \mathcal{F}_{2}$, and consequently, as $\underline{F}_{1} \in \mathcal{F}_{1}$, that $\mathcal{F}_{1} \succeq_{\mathrm{FSD}_{2}} \mathcal{F}_{2}$.

The proof of the second statement is similar.

When the lower and upper distributions of the p-box belong to our sets of distributions, they can be used to characterise our preferences between them. In that case, the stochastic dominance between two sets of cumulative distribution functions can be characterised by means of the relationships of stochastic dominance between their lower and upper distribution functions.

Corollary 1. Let $\mathcal{F}_{1}, \mathcal{F}_{2}$ be two sets of cumulative distribution functions, and let $\left(\underline{F}_{1}, \bar{F}_{1}\right)$ and $\left(\underline{F}_{2}, \bar{F}_{2}\right)$ denote their associated p-boxes. If $\underline{F}_{1}, \bar{F}_{1} \in \mathcal{F}_{1}$ and $\underline{F}_{2}, \bar{F}_{2} \in \mathcal{F}_{2}$, then

1. $\mathcal{F}_{1} \succeq_{\mathrm{FSD}_{1}} \mathcal{F}_{2} \Leftrightarrow \bar{F}_{1} \leq \underline{F}_{2}$.

2. $\mathcal{F}_{1} \succeq_{\mathrm{FSD}_{2}} \mathcal{F}_{2} \Leftrightarrow \mathcal{F}_{1} \succeq_{\mathrm{FSD}_{3}} \mathcal{F}_{2} \Leftrightarrow \underline{F}_{1} \leq \underline{F}_{2}$.

3. $\mathcal{F}_{1} \succeq_{\mathrm{FSD}_{4}} \mathcal{F}_{2} \Leftrightarrow \underline{F}_{1} \leq \bar{F}_{2}$.

4. $\mathcal{F}_{1} \succeq_{\mathrm{FSD}_{5}} \mathcal{F}_{2} \Leftrightarrow \mathcal{F}_{1} \succeq_{\mathrm{FSD}_{6}} \mathcal{F}_{2} \Leftrightarrow \bar{F}_{1} \leq \bar{F}_{2}$.

Proof. - If $\underline{F}_{2} \geq \underline{F}_{1} \in \mathcal{F}_{1}$, there is some $F_{1} \in \mathcal{F}_{1}$ such that $F_{1} \leq F_{2}$ for all $F_{2} \in \mathcal{F}_{2}$, and as a consequence $\mathcal{F}_{1} \succeq_{\mathrm{FSD}_{2}} \mathcal{F}_{2}$. 
- If $\underline{F}_{1} \in \mathcal{F}_{1}, \bar{F}_{2} \in \mathcal{F}_{2}$ and $\underline{F}_{1} \leq \bar{F}_{2}$, then there exist $F_{1} \in \mathcal{F}_{1}$ and $F_{2} \in \mathcal{F}_{2}$ such that $F_{1} \leq F_{2}$, whence $\mathcal{F}_{1} \succeq_{\mathrm{FSD}_{4}} \mathcal{F}_{2}$.

- If $\bar{F}_{1} \leq \bar{F}_{2}$, then since $\bar{F}_{2} \in \mathcal{F}_{2}$ then there is some $F_{2} \in \mathcal{F}_{2}$ such that $F_{1} \leq F_{2}$ for every $F_{1} \in \mathcal{F}_{1}$, because $F_{1} \leq \bar{F}_{1}$ for any $F_{1} \in \mathcal{F}_{1}$.

The remaining implications follow from Propositions 3 and 4 .

Remark 3 . The above corollary allows us to see more clearly the connection between the scenario proposed by Denoeux ([13]) and our proposal. Let $[U, V]$ and $\left[U^{\prime}, V^{\prime}\right]$ be two random closed intervals, whose associated belief and plausibility functions determine the sets of probability measures $\mathcal{P}, \mathcal{P}^{\prime}$. lences:

Applying Proposition 1 and Corollary 1, we obtain the following equiva-

- $\mathcal{P} \succeq_{\mathrm{FSD}_{1}} \mathcal{P}^{\prime} \Leftrightarrow F_{U}(t) \leq F_{V^{\prime}}(t)$ for every $t \in \mathbb{R} \Leftrightarrow \mathcal{P} \gg \mathcal{P}^{\prime}$.

- $\mathcal{P} \succeq_{\mathrm{FSD}_{2}} \mathcal{P}^{\prime} \Leftrightarrow \mathcal{P} \succeq_{\mathrm{FSD}_{3}} \mathcal{P}^{\prime} \Leftrightarrow F_{V}(t) \leq F_{V^{\prime}}(t)$ for every $t \in \mathbb{R} \Leftrightarrow \mathcal{P} \geqslant \mathcal{P}^{\prime}$.

- $\mathcal{P} \succeq_{\mathrm{FSD}_{4}} \mathcal{P}^{\prime} \Leftrightarrow F_{V}(t) \leq F_{U^{\prime}}(t)$ for every $t \in \mathbb{R} \Leftrightarrow \mathcal{P} \gtrsim \mathcal{P}^{\prime}$.

- $\mathcal{P} \succeq_{\mathrm{FSD}_{5}} \mathcal{P}^{\prime} \Leftrightarrow \mathcal{P} \succeq_{\mathrm{FSD}_{6}} \mathcal{P}^{\prime} \Leftrightarrow F_{U}(t) \leq F_{U^{\prime}}(t)$ for every $t \in \mathbb{R} \Leftrightarrow \mathcal{P}>\mathcal{P}^{\prime}$.

Hence, condition $>$ gives rise to $\left(F S D_{2}\right)$ (when $\mathcal{P}$ has a smallest distribution function) and $\left(F S D_{3}\right)$ (when it does not have it); similarly, condition $\geqslant$ produces $\left(F S D_{5}\right)$ (if $\mathcal{P}^{\prime}$ has a greatest distribution function) and $\left(F S D_{6}\right)$ (otherwise).

This also shows that our proposal is more general in the sense that it can be applied to arbitrary sets of probability measures, and not only those associated to a random closed interval. On the other hand, our work is more particular in the sense that we are assuming that our referential space is [0,1], instead of the real line. As we mentioned in the introduction, our results are immediately extendable to distribution functions taking values in any closed interval $[a, b]$, where $a<b$ are real numbers. The restriction to bounded intervals is made so that the lower envelope of a set of cumulative distribution functions is a finitely additive distribution function, which may not be the case if we consider the whole real line as our referential space. One solution to this problem is to add to our space a smallest and a greatest value $0_{\Omega}, 1_{\Omega}$, so that we always have $F\left(0_{\Omega}\right)=0$ and $F\left(1_{\Omega}\right)=1$.

Although in this paper we are focusing on sets of distribution functions associated to $\sigma$-additive probability measures, it is not uncommon to encounter situations where our imprecise information is given by means of sets of finitely additive probabilities: this is the case of the models of coherent lower and upper previsions in [48], and in particular of almost all models of non-additive measures considered in the literature [32]; in this sense they are easier to handle than sets of $\sigma$-additive probability measures, which do not have an easy characterisation in terms of their lower and upper envelopes, as showed in [27].

A finitely additive probability measure induces a finitely additive distribution function, and conversely, any finitely additive distribution function can be induced by a finitely additive probability measure [34]. As a consequence, given a $p$-box $(\underline{F}, \bar{F})$, the set of finitely additive probabilities compatible with this p-box induces the class of finitely additive distribution functions

$$
\mathcal{F}^{\prime}:=\{F \text { finitely additive distribution function }: \underline{F} \leq F \leq \bar{F}\} .
$$


In particular, both $\underline{F}, \bar{F}$ belong to $\mathcal{F}^{\prime}$. Taking this into account, if we define conditions of stochastic dominance for sets of finitely additive distribution functions analogous to those in Definition 4, it is not difficult to establish a characterisation similar to Corollary 1, whose analogous proof we omit:

Lemma 1. Let $\mathcal{F}_{1}^{\prime}, \mathcal{F}_{2}^{\prime}$ be two sets of finitely additive distribution functions with associated p-boxes $\left(\underline{F}_{1}, \bar{F}_{1}\right),\left(\underline{F}_{2}, \bar{F}_{2}\right)$. Assume $\underline{F}_{1}, \bar{F}_{1} \in \mathcal{F}_{1}^{\prime}$ and $\underline{F}_{2}, \bar{F}_{2} \in \mathcal{F}_{2}^{\prime}$.

1. $\mathcal{F}_{1}^{\prime} \succeq_{\mathrm{FSD}_{1}} \mathcal{F}_{2}^{\prime} \Leftrightarrow \bar{F}_{1} \leq \underline{F}_{2}$.

2. $\mathcal{F}_{1}^{\prime} \succeq_{\mathrm{FSD}_{2}} \mathcal{F}_{2}^{\prime} \Leftrightarrow \underline{F}_{1} \leq \underline{F}_{2}$.

3. $\mathcal{F}_{1}^{\prime} \succeq_{\mathrm{FSD}_{3}} \mathcal{F}_{2}^{\prime} \Leftrightarrow \underline{F}_{1} \leq \underline{F}_{2}$.

4. $\mathcal{F}_{1}^{\prime} \succeq_{\mathrm{FSD}_{4}} \mathcal{F}_{2}^{\prime} \Leftrightarrow \underline{F}_{1} \leq \bar{F}_{2}$.

5. $\mathcal{F}_{1}^{\prime} \succeq_{\mathrm{FSD}_{5}} \mathcal{F}_{2}^{\prime} \Leftrightarrow \bar{F}_{1} \leq \bar{F}_{2}$.

6. $\mathcal{F}_{1}^{\prime} \succeq_{\mathrm{FSD}_{6}} \mathcal{F}_{2}^{\prime} \Leftrightarrow \bar{F}_{1} \leq \bar{F}_{2}$.

Let $\mathcal{P}_{1}, \mathcal{P}_{2}$ be two sets of $\sigma$-additive probability measures, with associated p-boxes $\left(\underline{F}_{1}, \bar{F}_{1}\right),\left(\underline{F}_{2}, \bar{F}_{2}\right)$. Let $\mathcal{F}_{1}^{\prime}, \mathcal{F}_{2}^{\prime}$ be two sets of finitely additive distribution functions associated to the $p$-boxes $\left(\underline{F}_{1}, \bar{F}_{1}\right),\left(\underline{F}_{2}, \bar{F}_{2}\right)$. When the lower and upper distribution functions of the associated $p$-box belong to our set of cumulative distribution functions, we immediately deduce from Corollary 1 and Lemma 1 that stochastic dominance holds under the same conditions regardless of whether we work with finitely or $\sigma$-additive probability measures:

Proposition 5. Let us consider two sets of cumulative distribution functions $\mathcal{F}_{1}$ and $\mathcal{F}_{2}$ with associated p-boxes $\left(\underline{F}_{1}, \bar{F}_{1}\right),\left(\underline{F}_{2}, \bar{F}_{2}\right)$, and let $\mathcal{F}_{1}^{\prime}, \mathcal{F}_{2}^{\prime}$ be the sets of finitely additive distribution functions associated to these p-boxes. If $\underline{F}_{1}, \bar{F}_{1} \in \mathcal{F}_{1}$ and $\underline{F}_{2}, \bar{F}_{2} \in \mathcal{F}_{2}$, it holds that :

$$
\mathcal{F}_{1} \succeq_{\mathrm{FSD}_{\mathrm{i}}} \mathcal{F}_{2} \Leftrightarrow \mathcal{F}_{1}^{\prime} \succeq_{\mathrm{FSD}_{\mathrm{i}}} \mathcal{F}_{2}^{\prime},
$$

for $i=1, \ldots, 6$.

On the other hand, any finitely additive cdf $F$ can be approximated by a $\sigma$-additive cdf $\tilde{F}$ : its right-continuous approximation, given by

$$
\tilde{F}(x)=\inf _{y>x} F(y) \forall x<1, \tilde{F}(1)=1 .
$$

Hence, to any set $\mathcal{F}$ of finitely additive cdfs we can associate a set $\tilde{\mathcal{F}}$ of $\sigma$ additive cdfs, by considering $\tilde{\mathcal{F}}:=\{\tilde{F}: F \in \mathcal{F}\}$, and where $\tilde{F}$ is given by Eq. (4). However, both sets do not model the same preferences:

Proposition 6. Let $\mathcal{F}$ be a set of finitely additive cdfs, and let $\tilde{\mathcal{F}}$ be the set of their $\sigma$-additive approximations. The possible relationships between $\mathcal{F}$ and $\tilde{\mathcal{F}}$ are summarised in the following table:

\begin{tabular}{ccccccc}
\hline & $F S D_{1}$ & $F S D_{2}$ & $F S D_{3}$ & $F S D_{4}$ & $F S D_{5}$ & $F S D_{6}$ \\
\hline $\mathcal{F} \succ_{\mathrm{FSD}_{\mathrm{i}}} \tilde{\mathcal{F}}$ & $\bullet$ & $\bullet$ & $\bullet$ & $\bullet$ & $\bullet$ & $\bullet$ \\
\hline$\tilde{\mathcal{F}} \succ_{\mathrm{FSD}_{\mathrm{i}}} \mathcal{F}$ & & & & & & \\
\hline $\mathcal{F} \equiv_{\mathrm{FSD}_{\mathrm{i}}} \tilde{\mathcal{F}}$ & $\bullet$ & $\bullet$ & $\bullet$ & $\bullet$ & $\bullet$ & $\bullet$ \\
\hline $\mathcal{F}, \tilde{\mathcal{F}}$ incomparable & $\bullet$ & $\bullet$ & & & $\bullet$ & \\
\hline
\end{tabular}

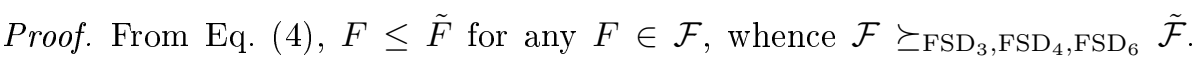
We deduce from Proposition 2 that we cannot have $\tilde{\mathcal{F}} \succ_{\mathrm{FSD}_{\mathrm{i}}} \mathcal{F}$ for any $i=$ $1, \ldots, 6$. To see that the other scenarios are possible, we refer to Example 3 in the Appendix. 


\section{Particular cases}

In this section, we investigate the stochastic dominance between sets of probability distributions in a number of particular cases of their associated p-boxes.

\subsection{Increasing imprecision}

We begin by studying the behaviour of the different notions of stochastic dominance for sets of distributions when we use them to compare two sets of distribution functions, one of which is more imprecise than the other. This may be useful in some situations: for instance, p-boxes can be seen as confidence bands [10, 39], which model our imprecise information about a distribution function taking into account a given sample and a fixed confidence level. Then if we apply two different confidence levels to the same data, we obtain two confidence bands, one included in the other, and we may study which of the two is preferred according to the different criteria we have proposed. In this sense, we may also study our preferences between a set of portfolios, that we represent by means of a set of distribution functions, and a greater set, where we include more distribution functions, but where also the associated risk may increase.

We are going to consider two different situations: the first one is when our information is given by a set of distribution functions. Hence, we consider two sets $\mathcal{F}_{1} \subseteq \mathcal{F}_{2}$ and investigate our preferences between them:

Proposition 7. Let us consider two sets of cumulative distribution functions $\mathcal{F}_{1}$ and $\mathcal{F}_{2}$ such that $\mathcal{F}_{1} \subseteq \mathcal{F}_{2}$. It holds that:

1. If $\mathcal{F}_{1}$ has only one distribution function, then all the possibilities are valid for $\left(F S D_{1}\right)$. Otherwise, if $\mathcal{F}_{1}$ is made by more than one distribution function, $\mathcal{F}_{1}$ and $\mathcal{F}_{2}$ are incomparable with respect to $\left(F S D_{1}\right)$.

2. With respect to $\left(F S D_{2}\right), \ldots,\left(F S D_{6}\right)$, the possible scenarios are summarised in the following table:

\begin{tabular}{cccccc}
\hline & $F S D_{2}$ & $F S D_{3}$ & $F S D_{4}$ & $F S D_{5}$ & $F S D_{6}$ \\
\hline $\mathcal{F}_{1} \succ_{\mathrm{FSD}_{\mathrm{i}}} \mathcal{F}_{2}$ & & & & $\bullet$ & $\bullet$ \\
\hline $\mathcal{F}_{2} \succ_{\mathrm{FSD}_{\mathrm{i}}} \mathcal{F}_{1}$ & $\bullet$ & $\bullet$ & & & \\
\hline $\mathcal{F}_{1} \equiv_{\mathrm{FSD}_{\mathrm{i}}} \mathcal{F}_{2}$ & $\bullet$ & $\bullet$ & $\bullet$ & $\bullet$ & $\bullet$ \\
\hline $\mathcal{F}_{1}, \mathcal{F}_{2}$ incomparable & $\bullet$ & & & $\bullet$ & \\
\hline
\end{tabular}

Proof. - On the one hand, if $\mathcal{F}_{1}$ has more than one cumulative distribution function, we deduce that $\mathcal{F}_{1}$ is incomparable with itself with respect to $\left(F S D_{1}\right)$, and as a consequence it is also incomparable with respect to the greater set $\mathcal{F}_{2}$.

- Since $\mathcal{F}_{1} \subseteq \mathcal{F}_{2}$, for any $F_{1} \in \mathcal{F}_{1}$ there exists $F_{2} \in \mathcal{F}_{2}$ such that $F_{1}=F_{2}$. Hence, we always have $\mathcal{F}_{2} \succeq_{\mathrm{FSD}_{3}} \mathcal{F}_{1}$ and $\mathcal{F}_{1} \succeq_{\mathrm{FSD}_{6}} \mathcal{F}_{2}$. Thus, we obtain that $\mathcal{F}_{1} \nsucc_{\mathrm{FSD}_{3}} \mathcal{F}_{2}, \mathcal{F}_{2} \nsucc_{\mathrm{FSD}_{6}} \mathcal{F}_{1}$, and both sets cannot be incomparable with respect to $\left(F S D_{3}\right)$ and $\left(F S D_{6}\right)$. Moreover, using Proposition 2 $\mathcal{F}_{1} \succ_{\mathrm{FSD}_{2}} \mathcal{F}_{2}$ and $\mathcal{F}_{2} \succ_{\mathrm{FSD}_{5}} \mathcal{F}_{1}$ are not possible. This also shows that $\mathcal{F}_{1} \equiv_{\mathrm{FSD}_{4}} \mathcal{F}_{2}$, because any $F \in \mathcal{F}_{1} \subseteq \mathcal{F}_{2}$ is equivalent to itself.

To see that all the other scenarios are indeed possible, we refer to Example 4 in the Appendix. 
Remark 4. A particular case of the above result would be when we compare a set of distribution functions $\mathcal{F}_{1}$ with itself, i.e., when $\mathcal{F}_{2}=\mathcal{F}_{1}$. In that case, $\mathcal{F}_{1} \equiv_{\mathrm{FSD}_{\mathrm{i}}} \mathcal{F}_{1}$ for $i=3,4,6$, as we have already noted in Remark 1 .

With respect to $\left(F S D_{1}\right),\left(F S D_{2}\right)$ and $\left(F S D_{5}\right)$, we may have either incomparability (for instance with $\mathcal{F}_{1}=\mathcal{F}_{2}=\left\{i d, I_{[0.5,1]}\right\}$ ) or indifference (with $\left.\mathcal{F}_{1}=\mathcal{F}_{2}=\{i d\}\right)$, but never strict preference.

The second scenario corresponds to the case where our information about the set of distribution functions is given by means of a p-box. A more imprecise p-box means that either the lower distribution function is smaller, the upper distribution function is greater, or both. We begin by considering the latter case.

Proposition 8. Let us consider two sets of cumulative distribution functions $\mathcal{F}_{1}$ and $\mathcal{F}_{2}$, and let $\left(\underline{F}_{1}, \bar{F}_{1}\right)$ and $\left(\underline{F}_{2}, \bar{F}_{2}\right)$ denote their associated p-boxes. Assume that $\underline{F}_{2}<\underline{F}_{1}<\bar{F}_{1}<\bar{F}_{2}$. Then the possible scenarios of stochastic dominance are summarised in the following table:

\begin{tabular}{ccccccc}
\hline & $F S D_{1}$ & $F S D_{2}$ & $F S D_{3}$ & $F S D_{4}$ & $F S D_{5}$ & $F S D_{6}$ \\
\hline $\mathcal{F}_{1} \succ_{\mathrm{FSD}_{\mathrm{i}}} \mathcal{F}_{2}$ & & & & $\bullet$ & $\bullet$ & $\bullet$ \\
\hline $\mathcal{F}_{2} \succ_{\mathrm{FSD}_{\mathrm{i}}} \mathcal{F}_{1}$ & & $\bullet$ & $\bullet$ & $\bullet$ & & \\
\hline $\mathcal{F}_{1} \equiv_{\mathrm{FSD}_{\mathrm{i}}} \mathcal{F}_{2}$ & & & & $\bullet$ & & \\
\hline $\mathcal{F}_{1}, \mathcal{F}_{2}$ incomparable & $\bullet$ & $\bullet$ & $\bullet$ & $\bullet$ & $\bullet$ & $\bullet$ \\
\hline
\end{tabular}

Proof. Using Proposition 2, we know that $\mathcal{F}_{1} \succeq_{\mathrm{FSD}_{1}} \mathcal{F}_{2}$ if and only if $\bar{F}_{1} \leq$ $\underline{F}_{2}$, which is incompatible with the assumptions. Similarly, we can see that $\mathcal{F}_{2} \nsucceq_{\mathrm{FSD}_{1}} \mathcal{F}_{1}$ and as a consequence they are incomparable.

On the other hand, if $\mathcal{F}_{1} \succeq_{\mathrm{FSD}_{\mathrm{i}}} \mathcal{F}_{2}$, for $i=2,3$, using Proposition 2 we would have that $\underline{F}_{1} \leq \underline{F}_{2}$, a contradiction with the hypothesis.

Similarly, if $\mathcal{F}_{2} \succeq_{\mathrm{FSD}_{\mathrm{i}}} \mathcal{F}_{1}$, for $i=5,6$, we deduce from Proposition 2 that $\bar{F}_{2} \leq \bar{F}_{1}$, again a contradiction.

To see that the scenarios included in the table are possible see Example 5 in the Appendix.

Although the inclusion $\mathcal{F}_{1} \subseteq \mathcal{F}_{2}$ implies that $\underline{F}_{2} \leq \underline{F}_{1} \leq \bar{F}_{1} \leq \bar{F}_{2}$, we may have $\underline{F}_{2}<\underline{F}_{1}<\bar{F}_{1}<\bar{F}_{2}$ even if $\mathcal{F}_{1}$ and $\overline{\mathcal{F}}_{2}$ are disjoint, for instance when these lower and upper distribution functions are $\sigma$-additive and we take $\mathcal{F}_{1}=\left\{\underline{F}_{1}, \bar{F}_{1}\right\}$ and $\mathcal{F}_{2}=\left\{\underline{F}_{2}, \bar{F}_{2}\right\}$. This is the reason why in Proposition 7 we cannot have $\mathcal{F}_{1} \succ_{\mathrm{FSD}_{4}} \mathcal{F}_{2}$ nor $\mathcal{F}_{2} \succ_{\mathrm{FSD}_{4}} \mathcal{F}_{1}$ and under the conditions of Proposition 8 we can.

Proposition 9. Under the conditions of Proposition 8, if in addition $\underline{F}_{1}, \bar{F}_{1} \in$ $\mathcal{F}_{1}$ and $\underline{F}_{2}, \bar{F}_{2} \in \mathcal{F}_{2}$, the possible scenarios are:

\begin{tabular}{ccccccc}
\hline & $F S D_{1}$ & $F S D_{2}$ & $F S D_{3}$ & $F S D_{4}$ & $F S D_{5}$ & $F S D_{6}$ \\
\hline $\mathcal{F}_{1} \succ_{\mathrm{FSD}_{\mathrm{i}}} \mathcal{F}_{2}$ & & & & & $\bullet$ & $\bullet$ \\
\hline $\mathcal{F}_{2} \succ_{\mathrm{FSD}_{\mathrm{i}}} \mathcal{F}_{1}$ & & $\bullet$ & $\bullet$ & & & \\
\hline $\mathcal{F}_{1} \equiv_{\mathrm{FSD}_{\mathrm{i}}} \mathcal{F}_{2}$ & & & & $\bullet$ & & \\
\hline $\mathcal{F}_{1}, \mathcal{F}_{2}$ incomparable & $\bullet$ & & & & & \\
\hline
\end{tabular}

Proof. - It is obvious that $\mathcal{F}_{1}$ and $\mathcal{F}_{2}$ are incomparable with respect to Definition $\left(F S D_{1}\right)$. 
- It holds that $\underline{F}_{2}<\underline{F}_{1} \leq F_{1}$ for every $F_{1} \in \mathcal{F}_{1}$, and consequently $\mathcal{F}_{2} \succ_{\mathrm{FSD}_{2}}$ $\mathcal{F}_{1}$. Moreover, using Corollary $1\left(F S D_{2}\right)$ and $\left(F S D_{3}\right)$ are equivalent, and consequently $\mathcal{F}_{2} \succ_{\mathrm{FSD}_{3}} \mathcal{F}_{1}$.

- Since $\underline{F}_{2}<\underline{F}_{1}$, it holds that $\mathcal{F}_{2} \succeq_{\mathrm{FSD}_{4}} \mathcal{F}_{1}$, and moreover $\bar{F}_{1}<\bar{F}_{2}$ implies that $\mathcal{F}_{1} \succeq_{\mathrm{FSD}_{4}} \mathcal{F}_{2}$. Thus, $\mathcal{F}_{1} \equiv_{\mathrm{FSD}_{4}} \mathcal{F}_{2}$.

- It holds that $F_{1} \leq \bar{F}_{1}<\bar{F}_{2}$ for every $F_{1} \in \mathcal{F}_{1}$, and consequently $\mathcal{F}_{1} \succ_{\mathrm{FSD}_{5}}$ $\mathcal{F}_{2}$. Furthermore, using Corollary $1,\left(F S D_{5}\right)$ and $\left(F S D_{6}\right)$ are equivalent, and consequently $\mathcal{F}_{1} \succ_{\mathrm{FSD}_{6}} \mathcal{F}_{2}$.

In particular, the above result is applicable when $\mathcal{F}_{1}=\left(\underline{F}_{1}, \bar{F}_{1}\right)$ and $\mathcal{F}_{2}=$ $\left(\underline{F}_{2}, \bar{F}_{2}\right)$, with $\underline{F}_{1}, \bar{F}_{1} \in \mathcal{F}_{1}$ and $\underline{F}_{2}, \bar{F}_{2} \in \mathcal{F}_{2}$.

To conclude this section, we consider the case where only one of the bounds becomes more imprecise in the second p-box.

Proposition 10. Let us consider two sets of cumulative distribution functions $\mathcal{F}_{1}$ and $\mathcal{F}_{2}$, and let $\left(\underline{F}_{1}, \bar{F}_{1}\right)$ and $\left(\underline{F}_{2}, \bar{F}_{2}\right)$ denote their associated p-boxes.

a) Assume that $\underline{F}_{2}<\underline{F}_{1}<\bar{F}_{1}=\bar{F}_{2}$. Then the possible scenarios are:

\begin{tabular}{ccccccc}
\hline & $F S D_{1}$ & $F S D_{2}$ & $F S D_{3}$ & $F S D_{4}$ & $F S D_{5}$ & $F S D_{6}$ \\
\hline $\mathcal{F}_{1} \succ_{\mathrm{FSD}_{\mathrm{i}}} \mathcal{F}_{2}$ & & & & $\bullet$ & $\bullet$ & $\bullet$ \\
\hline $\mathcal{F}_{2} \succ_{\mathrm{FSD}_{\mathrm{i}}} \mathcal{F}_{1}$ & & $\bullet$ & $\bullet$ & $\bullet$ & $\bullet$ & $\bullet$ \\
\hline $\mathcal{F}_{1} \equiv_{\mathrm{FSD}_{\mathrm{i}}} \mathcal{F}_{2}$ & & & & $\bullet$ & $\bullet$ & $\bullet$ \\
\hline $\mathcal{F}_{1}, \mathcal{F}_{2}$ incomparable & $\bullet$ & $\bullet$ & $\bullet$ & $\bullet$ & $\bullet$ & $\bullet$ \\
\hline
\end{tabular}

b) Assume that $\underline{F}_{2}=\underline{F}_{1}<\bar{F}_{1}<\bar{F}_{2}$. Then the possible situations are:

\begin{tabular}{ccccccc}
\hline & $F S D_{1}$ & $F S D_{2}$ & $F S D_{3}$ & $F S D_{4}$ & $F S D_{5}$ & $F S D_{6}$ \\
\hline $\mathcal{F}_{1} \succ_{\mathrm{FSD}_{\mathrm{i}}} \mathcal{F}_{2}$ & & $\bullet$ & $\bullet$ & $\bullet$ & & \\
\hline $\mathcal{F}_{2} \succ_{\mathrm{FSD}_{\mathrm{i}}} \mathcal{F}_{1}$ & & $\bullet$ & $\bullet$ & $\bullet$ & $\bullet$ & $\bullet$ \\
\hline $\mathcal{F}_{1} \equiv_{\mathrm{FSD}_{\mathrm{i}}} \mathcal{F}_{2}$ & & $\bullet$ & $\bullet$ & $\bullet$ & & \\
\hline $\mathcal{F}_{1}, \mathcal{F}_{2}$ incomparable & $\bullet$ & $\bullet$ & $\bullet$ & $\bullet$ & $\bullet$ & $\bullet$ \\
\hline
\end{tabular}

Proof. Let us prove the first statement; the proof of the second is analogous to this one. On the one hand, since $\bar{F}_{1}>\underline{F}_{2}$ and $\bar{F}_{2}>\underline{F}_{1}$, we deduce from Proposition 3 that $\mathcal{F}_{1}$ and $\mathcal{F}_{2}$ are incomparable with respect to $\left(F S D_{1}\right)$.

With respect to $\left(F S D_{2}\right),\left(F S D_{3}\right)$, note that if $\underline{F}_{2}<\underline{F}_{1}$, then there is some $x_{0} \in[0,1]$ such that $\underline{F}_{2}\left(x_{0}\right)=\inf _{F_{2} \in \mathcal{F}_{2}} F_{2}\left(x_{0}\right)<\underline{F}_{1}\left(x_{0}\right)$, whence there exists $F_{2}^{*} \in \mathcal{F}_{2}$ such that $F_{2}^{*}\left(x_{0}\right)<\underline{F}_{1}\left(x_{0}\right) \leq F_{1}\left(x_{0}\right)$ for all $F_{1} \in \mathcal{F}_{1}$. Thus, $F_{1} \not \leq F_{2}^{*}$ for any $F_{1} \in \mathcal{F}_{1}$ and $\mathcal{F}_{1} \succeq_{\mathrm{FSD}_{3}} \mathcal{F}_{2}$. Applying Proposition $2, \mathcal{F}_{1} \succeq_{\mathrm{FSD}_{2}} \mathcal{F}_{2}$.

In Example 6 of the Appendix we give examples showing that when the lower distribution function is smaller in the second p-box and the upper distribution functions coincide, all the possibilities not ruled out in the first table of the previous proposition can arise. Similar examples can be constructed for the case where $\underline{F}_{1}=\underline{F}_{2}$ and $\bar{F}_{1}<\bar{F}_{2}$.

\subsection{Sets of distribution functions associated to the same p-box}

Next we investigate the relationships between the preferences on two sets of distribution functions associated to the same p-box. We consider just the case of non-trivial p-boxes (that is, those where the lower and the upper distribution functions are different), since otherwise we obviously have indifference. 
Proposition 11. Let us consider two sets of cumulative distribution functions $\mathcal{F}_{1}$ and $\mathcal{F}_{2}$ such that $\underline{F}_{1}=\underline{F}_{2}, \bar{F}_{1}=\bar{F}_{2}$ and $\underline{F}_{1}<\bar{F}_{1}$. Then:

1. $\mathcal{F}_{1}$ and $\mathcal{F}_{2}$ are incomparable with respect to $\left(F S D_{1}\right)$.

2. With respect to $\left(F S D_{i}\right), i=2, \ldots, 6$, we may have incomparability, strict stochastic dominance or indifference between $\mathcal{F}_{1}$ and $\mathcal{F}_{2}$.

Proof. First of all, let us study the case of $\left(F S D_{1}\right)$. By Proposition $3, \mathcal{F}_{1} \succeq_{\mathrm{FSD}_{1}}$ $\mathcal{F}_{2}$ if and only if $\bar{F}_{1} \leq \underline{F}_{2}$, which in this case holds if and only if $\bar{F}_{1}=\underline{F}_{1}$, a contradiction with our hypotheses.

With respect to conditions $\left(F S D_{2}\right), \ldots,\left(F S D_{6}\right)$, it is easy to find examples of indifference by taking $\mathcal{F}_{1}=\mathcal{F}_{2}$ including the lower and upper distribution functions. To see that may also have strict dominance or incomparability, we refer to Example 7 in the Appendix.

When the lower and upper distribution functions belong to our set of distributions, Corollary 1 implies the following:

Corollary 2. Let us consider two sets of cumulative distribution functions $\mathcal{F}_{1}$ and $\mathcal{F}_{2}$ such that $\underline{F}_{1}=\underline{F}_{2}, \bar{F}_{1}=\bar{F}_{2}$ and $\underline{F}_{1}<\bar{F}_{1}$, and where $\underline{F}_{1}, \bar{F}_{1} \in \mathcal{F}_{1} \cap \mathcal{F}_{2}$. Then $\mathcal{F}_{1} \equiv_{\mathrm{FSD}_{\mathrm{i}}} \mathcal{F}_{2}$ for $i=2, \ldots, 6$, and they are incomparable with respect to $\left(F S D_{1}\right)$.

Next we investigate the case when we compare these two sets of distribution functions with a third one, and study if they produce the same preferences:

Proposition 12. Let $\mathcal{F}_{1}, \mathcal{F}_{1}^{*}$ and $\mathcal{F}_{2}$ be three sets of cumulative distribution functions such that $\underline{F}_{1}=\underline{F}_{1}^{*}$ and $\bar{F}_{1}^{*}=\bar{F}_{1}$. In that case:

1. $\mathcal{F}_{1} \succeq_{\mathrm{FSD}_{1}} \mathcal{F}_{2} \Leftrightarrow \mathcal{F}_{1}^{*} \succeq_{\mathrm{FSD}_{1}} \mathcal{F}_{2}$, and $\mathcal{F}_{2} \succeq_{\mathrm{FSD}_{1}} \mathcal{F}_{1} \Leftrightarrow \mathcal{F}_{2} \succeq_{\mathrm{FSD}_{1}} \mathcal{F}_{1}^{*}$.

2. Concerning definitions $\left(F S D_{2}\right), \ldots,\left(F S D_{6}\right)$, if we assume that $\mathcal{F}_{1} \succ_{\mathrm{FSD}_{\mathrm{i}}}$ $\mathcal{F}_{2}$, then the possible scenarios for the relationship between $\mathcal{F}_{1}^{*}$ and $\mathcal{F}_{2}$ are:

\begin{tabular}{cccccc}
\hline & $F S D_{2}$ & $F S D_{3}$ & $F S D_{4}$ & $F S D_{5}$ & $F S D_{6}$ \\
\hline $\mathcal{F}_{1}^{*} \succ_{\mathrm{FSD}_{\mathrm{i}}} \mathcal{F}_{2}$ & $\bullet$ & $\bullet$ & $\bullet$ & $\bullet$ & $\bullet$ \\
\hline $\mathcal{F}_{2} \succ_{\mathrm{FSD}_{\mathrm{i}}} \mathcal{F}_{1}^{*}$ & & $\bullet$ & $\bullet$ & & $\bullet$ \\
\hline $\mathcal{F}_{1}^{*} \equiv_{\mathrm{FSD}_{\mathrm{i}}} \mathcal{F}_{2}$ & & $\bullet$ & $\bullet$ & $\bullet$ & $\bullet$ \\
\hline $\mathcal{F}_{1}^{*}, \mathcal{F}_{2}$ incomparable & $\bullet$ & $\bullet$ & $\bullet$ & & $\bullet$ \\
\hline
\end{tabular}

Proof. Concerning definition $\left(F S D_{1}\right)$, it follows from Proposition 3 that $\mathcal{F}_{1} \succeq_{\mathrm{FSD}_{1}}$ $\mathcal{F}_{2}$ if and only if $\bar{F}_{1}^{*}=\bar{F}_{1} \leq \underline{F}_{2}$, and using the same result this is equivalent to $\mathcal{F}_{1}^{*} \succeq_{\mathrm{FSD}_{1}} \mathcal{F}_{2}$. The same result shows that $\mathcal{F}_{2} \succeq_{\mathrm{FSD}_{1}} \mathcal{F}_{1}$ if and only if $\bar{F}_{2} \leq \underline{F}_{1}=\underline{F}_{1}^{*}$, and this is again equivalent to $\mathcal{F}_{2} \succeq_{\text {FSD }} \mathcal{F}_{1}^{*}$.

Let us prove that $\mathcal{F}_{1} \succ_{\mathrm{FSD}_{2}} \mathcal{F}_{2}$ is not compatible with $\mathcal{F}_{2} \succeq_{\mathrm{FSD}_{2}} \mathcal{F}_{1}^{*}$. If $\mathcal{F}_{1} \succ_{\mathrm{FSD}_{2}} \mathcal{F}_{2}$, then $\mathcal{F}_{2} \succeq_{\mathrm{FSD}_{2}} \mathcal{F}_{1}$. This means that for every $F_{2} \in \mathcal{F}_{2}$ there exist $F_{1} \in \mathcal{F}_{1}$ and $x_{0}$ such that $F_{1}\left(x_{0}\right)<F_{2}\left(x_{0}\right)$. As a consequence,

$$
\inf _{F_{1}^{*} \in \mathcal{F}_{1}^{*}} F_{1}^{*}\left(x_{0}\right)=\underline{F}_{1}^{*}\left(x_{0}\right)=\underline{F}_{1}\left(x_{0}\right) \leq F_{1}\left(x_{0}\right)<F_{2}\left(x_{0}\right),
$$

whence for every $F_{2} \in \mathcal{F}_{2}$ there is some $F_{1}^{*} \in \mathcal{F}_{1}^{*}$ such that $F_{1}^{*}\left(x_{0}\right)<F_{2}\left(x_{0}\right)$, and consequently $F_{2} \not \leq F_{1}^{*}$. This means that $\mathcal{F}_{2} \nsucceq_{\mathrm{FSD}_{2}} \mathcal{F}_{1}^{*}$, and therefore we cannot have $\mathcal{F}_{2} \succ_{\mathrm{FSD}_{2}} \mathcal{F}_{1}^{*}$.

Let us show next that $\mathcal{F}_{1} \succ_{\mathrm{FSD}} \mathcal{F}_{2}$ implies that $\mathcal{F}_{1}^{*} \succeq_{\mathrm{FSD}} \mathcal{F}_{2}$. If $\mathcal{F}_{1} \succ_{\mathrm{FSD}}$ $\mathcal{F}_{2}$, there is $F_{2} \in \mathcal{F}_{2}$ such that $F_{1} \leq F_{2}$ for every $F_{1} \in \mathcal{F}_{1}$. Hence, $\bar{F}_{1} \leq F_{2}$, 
and therefore $\bar{F}_{1}^{*} \leq F_{2}$, which implies that also $F_{1}^{*} \leq F_{2}$ for every $F_{1}^{*} \in \mathcal{F}_{1}^{*}$. We conclude that $\mathcal{F}_{1}^{*} \succeq_{\mathrm{FSD}_{5}} \mathcal{F}_{2}$.

To see that the other scenarios are possible, see Example 8 in the Appendix.

Remark 5. Note that, under the conditions of the previous proposition, if we assume in addition that $\underline{F}_{1}, \bar{F}_{1} \in \mathcal{F}_{1} \cap \mathcal{F}_{1}^{*}$ and that $\underline{F}_{2}, \bar{F}_{2} \in \mathcal{F}_{2}$, then we deduce from Corollary 1 that $\mathcal{F}_{1} \succeq_{\mathrm{FSD}_{\mathrm{i}}} \mathcal{F}_{2} \Leftrightarrow \mathcal{F}_{1}^{*} \succeq_{\mathrm{FSD}_{\mathrm{i}}} \mathcal{F}_{2}$, for $i=1, \ldots, 6$.

\subsection{Convergence of p-boxes}

It is well-known that a distribution function can be seen as the limit of the empirical distribution function that we derive from a sample, as we increase the sample size. Something similar applies when we consider a set of distribution functions: it was proven in [35] that any p-box on the unit interval is the limit of a sequence of p-boxes $\left(\underline{F}_{n}, \bar{F}_{n}\right)_{n}$ that are discrete, in the sense that for every $n$ both $\underline{F}_{n}$ and $\bar{F}_{n}$ have a finite number of discontinuity points.

If for two given p-boxes $\left(\underline{F}_{1}, \bar{F}_{1}\right),\left(\underline{F}_{2}, \bar{F}_{2}\right)$ we consider respective approximating sequences $\left(\underline{F}_{1, n}, \bar{F}_{1, n}\right)_{n},\left(\underline{F}_{2, n}, \bar{F}_{2, n}\right)_{n}$, in the sense that

$$
\lim _{n} \underline{F}_{1, n}=\underline{F}_{1}, \lim _{n} \bar{F}_{1, n}=\bar{F}_{1}, \lim _{n} \underline{F}_{2, n}=\underline{F}_{2}, \lim _{n} \bar{F}_{2, n}=\bar{F}_{2},
$$

we may wonder if by comparing for each $n$ the discrete p-boxes $\left(\underline{F}_{1, n}, \bar{F}_{1, n}\right)$ and $\left(\underline{F}_{2, n}, \bar{F}_{2, n}\right)$ we can say something about the preferences between $\left(\underline{F}_{1}, \bar{F}_{1}\right)$ and $\left(\underline{F}_{2}, \bar{F}_{2}\right)$. This is what we set out to do in this section. We shall be even more general, by considering sets of distribution functions whose associated p-boxes converge to some limit.

Proposition 13. Let $\left(\mathcal{F}_{1, n}\right)_{n},\left(\mathcal{F}_{2, n}\right)_{n}$ be two sequences of sets of distribution functions and let us denote their associated sequences of p-boxes by $\left(\underline{F}_{1, n}, \bar{F}_{1, n}\right)$ and $\left(\underline{F}_{2, n}, \bar{F}_{2, n}\right)$ for $n \in \mathbb{N}$. Let $\mathcal{F}_{1}, \mathcal{F}_{2}$ be two sets of cumulative distribution functions with associated p-boxes $\left(\underline{F}_{1}, \bar{F}_{1}\right)$ and $\left(\underline{F}_{2}, \bar{F}_{2}\right)$. Assume that:

$$
\bar{F}_{1, n} \stackrel{n}{\rightarrow} \bar{F}_{1}, \underline{F}_{1, n} \stackrel{n}{\rightarrow} \underline{F}_{1}, \bar{F}_{2, n} \stackrel{n}{\rightarrow} \bar{F}_{2} \text { and } \underline{F}_{2, n} \stackrel{n}{\rightarrow} \underline{F}_{2},
$$

and that $\underline{F}_{1}, \bar{F}_{1} \in \mathcal{F}_{1}$ and $\underline{F}_{2}, \bar{F}_{2} \in \mathcal{F}_{2}$. Then, $\mathcal{F}_{1, n} \succeq_{\mathrm{FSD}_{\mathrm{i}}} \mathcal{F}_{2, n} \forall n$, implies that $\mathcal{F}_{1} \succeq_{\mathrm{FSD}_{\mathrm{i}}} \mathcal{F}_{2}$, for $i=1, \ldots, 6$.

Proof. The result follows from Proposition 3 and Corollary 1.

The assumption that the upper and lower distribution functions belong to the corresponding sets of distributions is not necessary for the implication with respect to $\left(F S D_{1}\right)$, but it is necessary for the other definitions.

\subsection{Stochastic dominance between possibility measures}

So far, we have explored the extension of the notion of stochastic dominance towards sets of probability measures, and we have showed that in some cases it is equivalent to compare the p-boxes they determine. There are some particular cases of sets of probability measures that can be summarised by means of a nonadditive measure, and in this way the work we have carried out connects with the theory of imprecise probabilities. Hence, we could use our results to compare two 
models of non-additive measures by comparing the sets of probability measures which are compatible with them. In this section, we consider the particular case where the sets of probability measures are those bounded by some given possibility measures.

Definition 5. [16] A possibility measure on [0,1] is a supremum preserving set function $\Pi: \mathcal{P}([0,1]) \rightarrow[0,1]$. It is characterised by its restriction to events $\pi$, which is called its possibility distribution. The conjugate function $N$ of a possibility measure is called a necessity measure.

Because of their computational simplicity, possibility measures are widely applied in many fields, including data analysis [46], diagnosis [9], cased-based reasoning [25], and psychology [40]. See [17] for an overview.

The connection between possibility measures and p-boxes was already explored in [47], and it was proven that almost any possibility measure can be seen as the natural extension of a corresponding p-box. However, the definition of this p-box implies defining some particular order on our referential space, which could be different to the one we already have there (for instance if the possibility measure is defined on $[0,1]$ it may seem counterintuitive to consider anything different from the natural order), and moreover two different possibility measures may produce two different orders on the same space, making it impossible to compare them.

Instead, we shall consider two possibility measures $\Pi_{1}$ and $\Pi_{2}$ on $\Omega=$ $[0,1]$, their associated sets of probability measures $\mathcal{M}\left(\Pi_{1}\right):=\{P$ probability : $\left.P(A) \leq \Pi_{1}(A) \forall A\right\}$, and $\mathcal{M}\left(\Pi_{2}\right):=\left\{P\right.$ probability $\left.: P(A) \leq \Pi_{2}(A) \forall A\right\}$ and the corresponding sets of distribution functions $\mathcal{F}_{1}$ and $\mathcal{F}_{2}$. Let $\left(\underline{F}_{1}, \bar{F}_{1}\right)$ and $\left(\underline{F}_{2}, \bar{F}_{2}\right)$ be their associated $p$-boxes.

Since any possibility measure on $[0,1]$ can be obtained as the upper probability of a random set [23], and moreover in that case [33] the upper probability of the random set is the maximum of the probability distributions of the measurable selections, we deduce that the $p$-boxes associated to $\mathcal{F}_{1}$ and $\mathcal{F}_{2}$ are determined by the following lower and upper distribution functions:

$$
\begin{aligned}
& \bar{F}_{1}(x)=\sup _{P \leq \Pi_{1}} P([0, x])=\Pi_{1}([0, x])=\sup _{y \leq x} \pi_{1}(y), \\
& \underline{F}_{1}(x)=\inf _{P \leq \Pi_{1}} P([0, x])=1-\Pi_{1}((x, 1])=1-\sup _{y>x} \pi_{1}(y)=N_{1}[0, x],
\end{aligned}
$$

and similarly

$$
\bar{F}_{2}(x)=\sup _{y \leq x} \pi_{2}(y)=\Pi_{2}[0, x] \text { and } \underline{F}_{2}(x)=1-\sup _{y>x} \pi_{2}(y)=N_{2}[0, x] .
$$

Note however, that these lower and upper distribution functions need not belong to $\mathcal{F}_{1}, \mathcal{F}_{2}$ : if for instance we consider the possibility measure associated to the possibility distribution $\pi=I_{(0.5,1]}$, we obtain $\bar{F}=\pi$, which is not right-continuous, and consequently cannot belong to the set $\mathcal{F}$ of distribution functions associated to $\mathcal{M}(\Pi)$.

In this section, we are going to use stochastic dominance to compare possibility measures associated to continuous distribution functions. In that case, both the lower and the upper distribution functions belong to the set of distribution functions associated to the possibility measures: 
Lemma 2. Let $\Pi$ be a possibility measure associated to a continuous possibility distribution on [0,1]. Then, there exist probability measures $P_{1}, P_{2} \in \mathcal{M}(\Pi)$ whose associated distribution functions are $F_{1}=\bar{F}, F_{2}=\underline{F}$.

Proof. Let us consider the probability space $\left([0,1], \beta_{[0,1]}, \lambda_{[0,1]}\right)$, where $\beta_{[0,1]}$ denotes the Borel $\sigma$-field and $\lambda_{[0,1]}$ the Lebesgue measure, and let $\Gamma:[0,1] \rightarrow$ $\mathcal{P}([0,1])$ be the random set given by $\Gamma(\alpha)=\{x: \pi(x) \geq \alpha\}=\pi^{-1}([\alpha, 1])$. It was proved in [23] that $\Pi$ is the upper probability of $\Gamma$.

Let us consider the mappings $U_{1}, U_{2}:[0,1] \rightarrow[0,1]$ given by $U_{1}(\alpha)=$ $\min \Gamma(\alpha), U_{2}(\alpha)=\max \Gamma(\alpha)$. Since we are assuming that $\pi$ is a continuous mapping, the set $\pi^{-1}([\alpha, 1])=\Gamma(\alpha)$ has a maximum and a minimum value for every $\alpha \in[0,1]$, so $U_{1}, U_{2}$ are well-defined. It also follows that $U_{1}, U_{2}$ are measurable mappings, and as a consequence the probability measures they induce $P_{U_{1}}, P_{U_{2}}$ belong to the set $\mathcal{M}(\Pi)$. Their associated distribution functions are:

$$
\begin{aligned}
F_{U_{1}}(x) & =P_{U_{1}}([0, x])=\lambda_{[0,1]}\left(U_{1}^{-1}([0, x])\right)=\lambda_{[0,1]}(\{\alpha: \min \Gamma(\alpha) \leq x\}) \\
& =\lambda_{[0,1]}(\{\alpha: \exists y \leq x: \pi(y) \geq \alpha\})=\lambda_{[0,1]}(\{\alpha: \Pi[0, x] \geq \alpha\}) \\
& =\Pi([0, x])=\bar{F}(x),
\end{aligned}
$$

where the fourth equality follows from the continuity of $\lambda_{[0,1]}$, and similarly

$$
\begin{aligned}
F_{U_{2}}(x) & =P_{U_{2}}([0, x])=\lambda_{[0,1]}\left(U_{2}^{-1}([0, x])\right)=\lambda_{[0,1]}(\{\alpha: \max \Gamma(\alpha) \leq x\}) \\
& =\lambda_{[0,1]}(\{\alpha: \pi(y)<\alpha \forall y>x\})=\lambda_{[0,1]}(\{\alpha: \Pi(x, 1] \leq \alpha\}) \\
& =1-\Pi((x, 1])=\underline{F}(x),
\end{aligned}
$$

again using the continuity of $\lambda_{[0,1]}$. Hence, $\bar{F}, \underline{F}$ belong to the set of distribution functions induced by $\mathcal{M}(\Pi)$.

As a consequence, if we consider two possibility measures $\Pi_{1}, \Pi_{2}$ with continuous possibility distributions $\pi_{1}, \pi_{2}$, the lower and upper distribution functions of their respective $p$-boxes belong to the sets $\mathcal{F}_{1}, \mathcal{F}_{2}$. Hence, we can apply Proposition 4 and conclude that $\mathcal{F}_{1} \succeq_{\mathrm{FSD}_{2}} \mathcal{F}_{2} \Leftrightarrow \mathcal{F}_{1} \succeq_{\mathrm{FSD}_{3}} \mathcal{F}_{2}$ and $\mathcal{F}_{1} \succeq_{\mathrm{FSD}_{5}} \mathcal{F}_{2} \Leftrightarrow \mathcal{F}_{1} \succeq_{\mathrm{FSD}_{6}} \mathcal{F}_{2}$. Moreover, we can use Corollary 1 and conclude that:

$$
\begin{aligned}
\mathcal{F}_{1} \succeq_{\mathrm{FSD}_{1}} \mathcal{F}_{2} \Leftrightarrow \bar{F}_{1} \leq \underline{F}_{2} \Leftrightarrow \Pi_{1}[0, x] \leq N_{2}[0, x] \forall x . \\
\mathcal{F}_{1} \succeq_{\mathrm{FSD}_{2}} \mathcal{F}_{2} \Leftrightarrow \underline{F}_{1} \leq \underline{F}_{2} \Leftrightarrow N_{1}[0, x] \leq N_{2}[0, x] \forall x \Leftrightarrow \Pi_{1}(x, 1] \geq \Pi_{2}(x, 1] \forall x . \\
\mathcal{F}_{1} \succeq_{\mathrm{FSD}_{4}} \mathcal{F}_{2} \Leftrightarrow \bar{F}_{1} \leq \bar{F}_{2} \Leftrightarrow N_{1}[0, x] \leq \Pi_{2}[0, x] \forall x . \\
\mathcal{F}_{1} \succeq_{\mathrm{FSD}_{5}} \mathcal{F}_{2} \Leftrightarrow \bar{F}_{1} \leq \bar{F}_{2} \Leftrightarrow \Pi_{1}[0, x] \leq \Pi_{2}[0, x] \forall x \Leftrightarrow N_{2}(x, 1] \leq N_{1}(x, 1] \forall x .
\end{aligned}
$$

From this we derive immediately the following result:

Proposition 14. Let $\mathcal{F}_{1}, \mathcal{F}_{2}$ be the sets of distribution functions associated to respective possibility measures $\Pi_{1}, \Pi_{2}$ with continuous possibility distributions.

1. $\Pi_{1} \leq N_{2} \Rightarrow \mathcal{F}_{1} \succeq_{\mathrm{FSD}_{1}} \mathcal{F}_{2}$.

2. $\Pi_{2} \leq \Pi_{1} \Rightarrow \mathcal{F}_{1} \succeq_{\mathrm{FSD}_{2}} \mathcal{F}_{2}, \mathcal{F}_{1} \succeq_{\mathrm{FSD}_{3}} \mathcal{F}_{2}$

3. $N_{1} \leq \Pi_{2} \Rightarrow \mathcal{F}_{1} \succeq_{\mathrm{FSD}_{4}} \mathcal{F}_{2}$.

4. $N_{2} \leq N_{1} \Rightarrow \mathcal{F}_{1} \succeq_{\mathrm{FSD}_{5}} \mathcal{F}_{2}, \mathcal{F}_{1} \succeq_{\mathrm{FSD}_{6}} \mathcal{F}_{2}$.

It is not difficult to show that none of the above sufficient conditions is necessary. 


\subsection{P-boxes where one of the bounds is trivial}

Let us investigate next the case of p-boxes where one of the bounds is trivial. These have been related to possibility and maxitive measures in [47], when working with finitely additive cdfs. It is not difficult to show that when the lower distribution function is trivial, the probability measures determined by the pbox are those dominated by the possibility measure that has $\bar{F}$ as a possibility distribution; however, a similar result does not hold for the case of $(\underline{F}, 1)$ in general, because we need $\underline{F}$ to be right-continuous.

Let us show that when the lower distribution function is trivial, then the second and third conditions, which are based on the comparison of this bound, always produce indifference.

Proposition 15. Let us consider the p-boxes $\mathcal{F}_{1}=\left(\underline{F}_{1}, \bar{F}_{1}\right)$ and $\mathcal{F}_{2}=\left(\underline{F}_{2}, \bar{F}_{2}\right)$. Let us assume that $\underline{F}_{1}=\underline{F}_{2}=I_{\{1\}}, \underline{F}_{1} \neq \bar{F}_{1}$ and $\underline{F}_{2} \neq \bar{F}_{2}$. Then the possible relationships between $\mathcal{F}_{1}$ and $\mathcal{F}_{2}$ are:

\begin{tabular}{ccccccc}
\hline & $F S D_{1}$ & $F S D_{2}$ & $F S D_{3}$ & $F S D_{4}$ & $F S D_{5}$ & $F S D_{6}$ \\
\hline $\mathcal{F}_{1} \succ_{\mathrm{FSD}_{\mathrm{i}}} \mathcal{F}_{2}$ & & & & & $\bullet$ & $\bullet$ \\
\hline $\mathcal{F}_{2} \succ_{\mathrm{FSD}_{\mathrm{i}}} \mathcal{F}_{1}$ & & & & & $\bullet$ & $\bullet$ \\
\hline $\mathcal{F}_{1} \equiv_{\mathrm{FSD}_{\mathrm{i}}} \mathcal{F}_{2}$ & & $\bullet$ & $\bullet$ & $\bullet$ & $\bullet$ & $\bullet$ \\
\hline $\mathcal{F}_{1}, \mathcal{F}_{2}$ incomparable & $\bullet$ & & & & $\bullet$ & $\bullet$ \\
\hline
\end{tabular}

Proof. - Using Proposition 3 we know that $\mathcal{F}_{1} \succeq_{\mathrm{FSD}_{1}} \mathcal{F}_{2} \Leftrightarrow \bar{F}_{1} \leq \underline{F}_{2}$, and this condition cannot hold because $\underline{F}_{2}=I_{\{1\}}$ and the $p$-boxes are not trivial. Consequently, both sets are incomparable with respect to $\left(F S D_{1}\right)$.

- Since $\underline{F}_{1}=\underline{F}_{2} \in \mathcal{F}_{1} \cap \mathcal{F}_{2}$, we deduce from Corollary 1 that $\mathcal{F}_{1} \equiv_{\mathrm{FSD}_{2}} \mathcal{F}_{2}$. Applying Proposition 2, we deduce that $\mathcal{F}_{1} \equiv_{\mathrm{FSD}_{3}} \mathcal{F}_{2}$ and $\mathcal{F}_{1} \equiv_{\mathrm{FSD}_{4}} \mathcal{F}_{2}$.

- On the other hand, it is easy to see that anything can happen for definitions $\left(F S D_{5}\right)$ and $\left(F S D_{6}\right)$, since these depend on the upper cumulative distribution functions of the p-boxes, on which no assumptions are made.

Similarly, when the upper distribution function is trivial, then the fifth and sixth conditions, which are based on the comparison of these bounds, always produce indifference. The proof is similar and therefore omitted.

Proposition 16. Let us consider the p-boxes $\mathcal{F}_{1}=\left(\underline{F}_{1}, \bar{F}_{1}\right)$ and $\mathcal{F}_{2}=\left(\underline{F}_{2}, \bar{F}_{2}\right)$. Let us assume that $\bar{F}_{1}=\bar{F}_{2}=1, \underline{F}_{1}<\bar{F}_{1}$ and $\underline{F}_{2}<\bar{F}_{2}$. Then the possible relationships between $\mathcal{F}_{1}$ and $\mathcal{F}_{2}$ are:

\begin{tabular}{ccccccc}
\hline & $F S D_{1}$ & $F S D_{2}$ & $F S D_{3}$ & $F S D_{4}$ & $F S D_{5}$ & $F S D_{6}$ \\
\hline $\mathcal{F}_{1} \succ_{\mathrm{FSD}_{\mathrm{i}}} \mathcal{F}_{2}$ & & $\bullet$ & $\bullet$ & & & \\
\hline $\mathcal{F}_{2} \succ_{\mathrm{FSD}_{\mathrm{i}}} \mathcal{F}_{1}$ & & $\bullet$ & $\bullet$ & & & \\
\hline $\mathcal{F}_{1} \equiv_{\mathrm{FSD}_{\mathrm{i}}} \mathcal{F}_{2}$ & & $\bullet$ & $\bullet$ & $\bullet$ & $\bullet$ & $\bullet$ \\
\hline $\mathcal{F}_{1}, \mathcal{F}_{2}$ incomparable & $\bullet$ & $\bullet$ & $\bullet$ & & & \\
\hline
\end{tabular}

\subsection{0-1-valued p-boxes}

To conclude this section, let us focus on 0-1-valued p-boxes, by which we mean p-boxes where both the lower and upper cumulative distribution functions 
$\underline{F}, \bar{F}$ are 0 -1-valued. They have also been related to possibility measures in [47]. Given a $0-1$-valued distribution function $F$, we denote

$$
x_{F}=\inf \{x \mid F(x)=1\} .
$$

Note that this infimum is a minimum when we consider distribution functions associated to $\sigma$-additive probability measures, but not necessarily for those associated to finitely additive probability measures.

Given a p-box $(\underline{F}, \bar{F})$, where both $\underline{F}, \bar{F}$ are 0 -1-valued, these two functions determine an interval $S$ such that $\left(x_{\bar{F}}, x_{F}\right) \subseteq S \subseteq\left[x_{\bar{F}}, x_{F}\right]$; the limit $x_{\bar{F}}$ is included in $S$ depending on whether $\bar{F}\left(x_{\bar{F}}\right)=1$ or not (and similarly for $x_{F}$ ). It is not difficult to show that the $p$-box $(\underline{F}, \bar{F})$ is equivalent to the set of probability measures that satisfy $P(S)=1$. Now, using the relationships between the conditions $\left(F S D_{1}\right), \ldots,\left(F S D_{6}\right)$ and the orderings between the interval of the real line, we can establish the following result:

Proposition 17. Let $\mathcal{F}_{1}$ and $\mathcal{F}_{2}$ be two sets of cumulative distribution functions, with associated p-boxes $\left(\underline{F}_{1}, \bar{F}_{1}\right),\left(\underline{F}_{2}, \bar{F}_{2}\right)$.

a) If $\underline{F}_{1}, \bar{F}_{1}, \underline{F}_{2}$ and $\bar{F}_{2}$ are 0-1-valued functions, then

1. $\mathcal{F}_{1} \succeq_{\mathrm{FSD}_{1}} \mathcal{F}_{2} \Leftrightarrow x_{\bar{F}_{1}} \geq x_{\underline{F}_{2}}$.

2. $\mathcal{F}_{1} \succeq_{\mathrm{FSD}_{2}} \mathcal{F}_{2} \Rightarrow x_{\underline{F}_{1}} \geq x_{\underline{F}_{2}}$.

3. $\mathcal{F}_{1} \succeq_{\mathrm{FSD}_{3}} \mathcal{F}_{2} \Rightarrow x_{\underline{F}_{1}} \geq x_{\underline{F}_{2}}$.

4. $\mathcal{F}_{1} \succeq_{\mathrm{FSD}_{4}} \mathcal{F}_{2} \Rightarrow x_{\underline{F}_{1}} \geq x_{\bar{F}_{2}}$.

5. $\mathcal{F}_{1} \succeq_{\mathrm{FSD}_{5}} \mathcal{F}_{2} \Rightarrow x_{\bar{F}_{1}} \geq x_{\bar{F}_{2}}$.

6. $\mathcal{F}_{1} \succeq_{\mathrm{FSD}_{6}} \mathcal{F}_{2} \Rightarrow x_{\bar{F}_{1}} \geq x_{\bar{F}_{2}}$.

Moreover, if $\underline{F}_{1}, \bar{F}_{1} \in \mathcal{F}_{1}$ and $\underline{F}_{2}, \bar{F}_{2} \in \mathcal{F}_{2}$, the converses also hold.

b) If in particular $\mathcal{F}_{1}$ and $\mathcal{F}_{2}$ are two sets of 0-1 cumulative distribution functions it also holds that

2. $x_{F_{1}}>x_{F_{2}} \Rightarrow \mathcal{F}_{1} \succeq_{\mathrm{FSD}_{2}} \mathcal{F}_{2}$.

3. $x_{\underline{F}_{1}}>x_{\underline{F}_{2}} \Rightarrow \mathcal{F}_{1} \succeq_{\mathrm{FSD}_{3}} \mathcal{F}_{2}$.

4. $x_{\underline{F}_{1}}>x_{\bar{F}_{2}} \Rightarrow \mathcal{F}_{1} \succeq_{\mathrm{FSD}_{4}} \mathcal{F}_{2}$

5. $x_{\bar{F}_{1}}>x_{\bar{F}_{2}} \Rightarrow \mathcal{F}_{1} \succeq_{\mathrm{FSD}_{5}} \mathcal{F}_{2}$.

6. $x_{\bar{F}_{1}}>x_{\bar{F}_{2}} \Rightarrow \mathcal{F}_{1} \succeq_{\mathrm{FSD}_{6}} \mathcal{F}_{2}$

Proof. In order to prove the first item of this result it is enough to consider Proposition 3, and to note that, if $F$ and $G$ are two $0-1$ finitely additive distribution functions then $F \leq G$ implies that $x_{F} \geq x_{G}$. In particular, if $G$ is a cumulative distribution function, $F \leq G$ if and only if $x_{F} \geq x_{G}$, from which we deduce that $x_{\bar{F}_{1}} \geq x_{\underline{F}_{2}} \Rightarrow \mathcal{F}_{1} \succeq_{\mathrm{FSD}_{1}} \mathcal{F}_{2}$. Moreover, if $\underline{F}_{1}, \bar{F}_{1} \in \mathcal{F}_{1}$ and $\underline{F}_{2}, \bar{F}_{2} \in \mathcal{F}_{2}$, these are cumulative distribution functions, and we can use that $F \leq G$ if and only if $x_{F} \geq x_{G}$. Applying Corollary 1 we deduce that in that case the converse implications also hold.

Let us consider the second part.

(2) If $x_{\underline{F}_{1}}>x_{\underline{F}_{2}}, \exists x_{0}$ such that $x_{\underline{F}_{1}}>x_{0}>x_{\underline{F}_{2}}$. Then, since $x_{0}>x_{\underline{F}_{2}}$, $\underline{F}_{2}\left(x_{0}\right)=1$ and therefore $F_{2}\left(x_{0}\right)=1, \forall F_{2} \in \mathcal{F}_{2}$. Since $x_{\underline{F}_{1}}>x_{0}, \underline{F}_{1}\left(x_{0}\right)=$ 0 and as we are considering only $0-1$ valued cdf, there is some $F_{1} \in \mathcal{F}_{1}$ such that $F_{1}\left(x_{0}\right)=0$. Thus, there is some $F_{1} \in \mathcal{F}_{1}$ s.t. $F_{1} \leq F_{2} \quad \forall F_{2} \in$ $\mathcal{F}_{2}$. 
(4) If $x_{\underline{F}_{1}}>x_{\bar{F}_{2}}, \exists x_{0}$ such that $x_{\underline{F}_{1}}>x_{0}>x_{\bar{F}_{2}}$. Then, $\bar{F}_{2}\left(x_{0}\right)=1$, and since all the cdf are $0-1$ valued, $\exists F_{2} \in \mathcal{F}_{2}$ such that $F_{2}\left(x_{0}\right)=1$. On the other hand, $\underline{F}_{1}\left(x_{0}\right)=0$, and since all the cdf are $0-1$ valued, there is some $F_{1} \in \mathcal{F}_{1}$ such that $F_{1}\left(x_{0}\right)=0$. Hence, $F_{1} \leq F_{2}$ and therefore $\mathcal{F}_{1} \succeq_{\mathrm{FSD}_{4}} \mathcal{F}_{2}$.

(5) If $x_{\bar{F}_{1}}>x_{\bar{F}_{2}}$, there is some $x_{0}$ such that $x_{\bar{F}_{1}}>x_{0}>x_{\bar{F}_{2}}$. Hence, $\bar{F}_{2}\left(x_{0}\right)=1$. Since all the cumulative distribution functions are $0-1$ valued, $\exists F_{2} \in \mathcal{F}_{2}$ such that $F_{2}\left(x_{0}\right)=1$. On the other hand, $\bar{F}_{1}\left(x_{0}\right)=0$, whence $F_{1}\left(x_{0}\right)=0$ for all $F_{1} \in \mathcal{F}_{1}$. Hence, $F_{2} \geq F_{1}$ for all $F_{1} \in \mathcal{F}_{1}$.

(3),(6) These follow from items (2),(5), respectively, and Proposition 2.

In Example 9 in the Appendix we show that the converse implications do not hold in general.

\section{Illustrative example: comparison of Lorenz curves}

As we mentioned in the introduction, the notion of stochastic dominance has been applied in many different contexts. One of the most interesting is in the field of social welfare [2, 31, 45], for comparing Lorenz curves. They are a graphical representation of the cumulative distribution function of the wealth: the elements of the population are ordered according to it, and the curve shows, for the bottom $\mathrm{x} \%$ elements, what percentage $\mathrm{y} \%$ of the total wealth they have. Hence, the Lorenz curve can be used as a measure of equality: the closest the curve is to the straight line, the more equal the associated society is.

If we have the Lorenz curves of two different countries, we can compare them by means of stochastic dominance: if one of them is dominated by the other, the closest to the straight line will be associated to a more equal society, and will therefore be considered preferable. In this section, we are going to use our extensions of stochastic dominance to compare sets of Lorenz curves associated to countries in different areas of the world. We shall consider the Lorenz curves associated to the quintiles of the empirical distribution functions. The following table provides the wealth in each of the quintiles (Source data: World Bank database. http://timetric.com/dataset/worldbank):

\begin{tabular}{cccccc}
\hline Country-year & $\mathbf{0 - 0 . 2}$ & $\mathbf{0 . 2 - 0 . 4}$ & $\mathbf{0 . 4 - 0 . 6}$ & $\mathbf{0 . 6 - 0 . 8}$ & $\mathbf{0 . 8 - 1}$ \\
\hline Australia-1994 & 5.9 & 12.01 & 17.2 & 23.57 & 41.32 \\
\hline Canada-2000 & 7.2 & 12.73 & 17.18 & 22.95 & 39.94 \\
\hline China-2005 & 5.73 & 9.8 & 14.66 & 22 & 47.81 \\
\hline Finland-2000 & 9.62 & 14.07 & 17.47 & 22.14 & 36.7 \\
\hline FYR Macedonia-2000 & 9.02 & 13.45 & 17.49 & 22.61 & 37.43 \\
\hline Greece-2000 & 6.74 & 11.89 & 16.84 & 23.04 & 41.49 \\
\hline India-2005 & 8.08 & 11.27 & 14.94 & 20.37 & 45.34 \\
\hline Japan-1993 & 10.58 & 14.21 & 17.58 & 21.98 & 35.65 \\
\hline Maldives-2004 & 6.51 & 10.88 & 15.71 & 22.66 & 44.24 \\
\hline Norway-2000 & 9.59 & 13.96 & 17.24 & 21.98 & 37.23 \\
\hline Sweden-2000 & 9.12 & 13.98 & 17.57 & 22.7 & 36.63 \\
\hline USA-2000 & 5.44 & 10.68 & 15.66 & 22.4 & 45.82 \\
\hline
\end{tabular}


To make the comparison by means of the extensions of stochastic dominance clearer, we are going to consider the cumulative distribution from the richest to the poorest group: in this way, we will always obtain a curve which is above the straight line, and it will comply with our idea of considering preferable the smallest distribution function. If we apply this to the data in the above table, we obtain the following:

\begin{tabular}{cccccc}
\hline Country-year & $\mathbf{F ( 0 . 2 )}$ & $\mathbf{F ( 0 . 4 )}$ & $\mathbf{F ( 0 . 6 )}$ & $\mathbf{F ( 0 . 8 )}$ & $\mathbf{F ( 1 )}$ \\
\hline Australia-1994 & 41.32 & 64.89 & 82.09 & 94.1 & 100 \\
\hline Canada-2000 & 39.94 & 62.89 & 80.07 & 92.8 & 100 \\
\hline China-2005 & 47.81 & 69.81 & 84.47 & 94.27 & 100 \\
\hline Finland-2000 & 36.7 & 58.84 & 76.31 & 90.38 & 100 \\
\hline FYR Macedonia-2000 & 37.43 & 60.04 & 77.53 & 90.98 & 100 \\
\hline Greece-2000 & 41.49 & 64.53 & 81.37 & 93.26 & 100 \\
\hline India-2005 & 45.34 & 65.71 & 80.65 & 91.92 & 100 \\
\hline Japan-1993 & 35.65 & 57.63 & 75.21 & 89.42 & 100 \\
\hline Maldives-2004 & 44.24 & 66.9 & 82.61 & 93.49 & 100 \\
\hline Norway-2000 & 37.23 & 59.21 & 76.45 & 90.41 & 100 \\
\hline Sweden-2000 & 36.63 & 59.33 & 76.9 & 90.88 & 100 \\
\hline USA-2000 & 45.82 & 68.22 & 83.88 & 94.56 & 100 \\
\hline
\end{tabular}

We are going to group these countries by continents/regions:

- Group 1: China, Japan, India.

- Group 2: Finland, Norway, Sweden.

- Group 3: Canada, USA.

- Group 4: FYR Macedonia, Greece.

- Group 5: Australia, Maldives.

The relationships between these groups are summarised in the following table:

\begin{tabular}{lccccc}
\hline & Group 1 & Group 2 & Group 3 & Group 4 & Group 5 \\
\hline Group 1 & $\equiv_{\mathrm{FSD}_{2,5}}$ & $\succ_{\mathrm{FSD}_{2}}$ & $\succ_{\mathrm{FSD}_{2}}$ & $\succ_{\mathrm{FSD}_{2}}$ & $\succ_{\mathrm{FSD}_{2}}$ \\
Group 2 & $\succ_{\mathrm{FSD}_{5}}$ & $\equiv_{\mathrm{FSD}_{3,6}}$ & $\succ_{\mathrm{FSD}_{1}}$ & $\succ_{\mathrm{FSD}_{1}}$ & $\succ_{\mathrm{FSD}_{1}}$ \\
Group 3 & $\equiv_{\mathrm{FSD}_{4}}$ & & ${ }_{\mathrm{FSD}_{2,5}}$ & $\succ_{\mathrm{FSD}_{2}}$ & $\succ_{\mathrm{FSD}_{2}}$ \\
Group 4 & $\succ_{\mathrm{FSD}_{5}}$ & & $\succ_{\mathrm{FSD}_{5}}$ & $\equiv_{\mathrm{FSD}_{3,6}}$ & $\succ_{\mathrm{FSD}_{3,6}}$ \\
Group 5 & $\succ_{\mathrm{FSD}_{5}}$ & & $\succ_{\mathrm{FSD}_{5}}$ & & $\equiv_{\mathrm{FSD}_{3,6}}$ \\
\hline
\end{tabular}

This means for instance that the set of distribution functions in the first group strictly dominate those in the second group according to definition $\left(F S D_{2}\right)$, and those in the second group strictly dominate those in the first group according to definition $\left(F S D_{5}\right)$. This is because the best country in the first group (Japan) stochastically dominates all the countries in the second group, but the worst (China) is stochastically dominated by all countries in the second group. This, together with Proposition 2, implies that the first group strictly dominates the second according to $\left(F S D_{3}\right)$, is strictly dominated by the second according to $\left(F S D_{6}\right)$, that they are indifferent according to $\left(F S D_{4}\right)$ and incomparable according to $\left(F S D_{1}\right)$. 
Similar considerations hold for the other pairwise comparisons. For instance, group 4 strictly dominates group 5 according to $\left(F S D_{3}\right),\left(F S D_{6}\right)$, but it does not dominate it according to $\left(F S D_{2}\right),\left(F S D_{5}\right)$. This also shows that conditions $\left(F S D_{2}\right)$ and $\left(F S D_{3}\right)$ are not equivalent (and similarly for $\left(F S D_{5}\right)$ and $\left.\left(F S D_{6}\right)\right)$.

The cells where we have left a blank space mean that no dominance relationship is satisfied: for instance, group 3 does not dominate group 2 according to any of the definitions.

Since all the groups have more than one element, they will not satisfy $\left(F S D_{1}\right)$ when comparing them to themselves. It follows from Remark 4 that they are always indifferent to themselves according to $\left(F S D_{3}\right),\left(F S D_{4}\right)$ and $\left(F S D_{6}\right)$; they are indifferent to themselves according to $\left(F S D_{2}\right)$ when they have a best-case-scenario (as it is the case for groups 1 and 3 ), and indifferent according to $\left(F S D_{5}\right)$ when they have a worst-case scenario (as it is the case again for groups 1 and 3), and incomparable according to these definitions in the other cases.

Note that we can also use the above data to illustrate some of the results in this paper: for instance, we saw in Remark 1 that condition $\left(F S D_{2}\right)$ is transitive, and in the table above we see that group 1 is preferred to group 3 according to $\left(F S D_{2}\right)$ and group 3 is preferred to group 4 according to $\left(F S D_{2}\right)$ : this allows us to infer immediately that group 1 is preferred to group 4 according to this condition. The comparison of the first two groups is an instance of Proposition 8 , because the p-box induced by the first group is strictly more imprecise (i.e., it has a smaller lower cdf and a greater upper cdf) than that of the second group.

\section{Conclusions}

In this paper we have extended the notion of stochastic dominance to the case where we compare pairs of sets of probability measures. We have proposed a number of definitions, related to the possible orderings we can establish between two intervals, and we have studied their properties in a number of scenarios, such as when we compare a set of probability measures with a more imprecise one or when we consider two sets of probability measures with the same lower and upper bounds.

We see that it is useful to represent our sets of probability measures by means of p-boxes, and that in some cases we can easily derive the relationship between the sets by looking at the lower and upper distribution functions of the associated p-box. This representation is useful when our set of distribution functions include the lower and upper distribution functions, as we can see by comparing Proposition 3 with Corollary 1 and Propositions 8 and 9. Although the situation is simpler when these limits are included, because our definitions reduce to Denoeux' ones based on orderings in the real line, this condition is not always satisfied, as we can see from Section 5 and the Appendix.

We have applied our results to the case where we want to compare two possibility measures by means of their associated sets of probabilities; an open problem would be to generalise this to the comparison of other models of nonadditive measures, such as $n$-monotone capacities and coherent lower previsions. For this, we have to deal carefully with the distinction between the finitely additive and the $\sigma$-additive probabilities subjacent to an imprecise model, since, 
as we have showed in Section 3, they may have different implications. Another open problem would be the generalisation of our results to other referential spaces.

Finally, an important generalisation of our work could be done towards the notion of 2- and, more generally, n-stochastic dominance, which also play an important role in the context of social welfare $[2,6,41]$.

\section{Acknowledgements}

The research in this paper has been partly supported by the project MTM201017844, and by the Science and Education Ministry FPU grant AP2009-1034. We would like to thank Susana Díaz, the anonymous reviewers and the guest editors for some helpful comments.

\section{References}

[1] V. Antoine, B. Quost, M.-H. Masson and T. Denoeux. CECM: Constrained evidential C-means algorithm. Computational Statistics and Data Analysis, $56(4): 948-968,2012$.

[2] B. Arnold. Majorization and the Lorenz Order: A Brief Introduction. Springer, 1987.

[3] Ph. Artzner, F. Delbaen, J.-M. Eber, and D. Heath. Coherent measures of risk. Mathematical Finance, 9:203-228, 1999.

[4] A. Atkinson. On the measurement of poverty. Econometrica, 55:749-764, 1987.

[5] C. Baudrit and D. Dubois. Practical representations of incomplete probabilistic knowledge. Computational Statistics and Data Analysis, 51(1):86$108,2006$.

[6] N. Bäuerle and M. Müller. Stochastic orders and risk measures: consistency and bounds. Insurance: Mathematics and Economics, 38(1):132-148, 2006.

[7] V. S. Bawa. Optimal rules for ordering uncertain prospects. Journal of Financial Economics, 2(1):95-121, 1975.

[8] J. O. Berger. Statistical Decision Theory and Bayesian Analysis. SpringerVerlag, New York, 1985.

[9] D. Cayrac, D. Dubois, M. Haziza, and H. Prade. Handling uncertainty with possibility theory and fuzzy sets in a satellite fault diagnosis application. IEEE Transactions on Fuzzy Systems, 4:251-259, 1996.

[10] R. Cheng, B. Evans, and J. Williams. Confidence band estimations for distributions used in probabilistic design. International Journal of Mechanical Sciences, 30:835-845, 1988.

[11] G. Choquet. Theory of capacities. Annales de l'Institut Fourier, 5:131-295, 1953-1954. 
[12] A. P. Dempster. Upper and lower probabilities generated by a random closed interval. Annals of Mathematical Statistics, 39:957-966, 1968.

[13] T. Denoeux. Extending stochastic ordering to belief functions on the real line. Information Sciences, 179:1362-1376, 2009.

[14] M. Denuit, J. Dhaene, M. Goovaerts, and R. Kaas. Actuarial theory for dependent risks. John Wiley \& Sons, 2005.

[15] S. Destercke, D. Dubois, and E. Chojnacki. Unifying practical representations- i: generalized p-boxes. International Journal of Approximate Reasoning, 49(3):649-663, 2008.

[16] D. Dubois and H. Prade. Possibility Theory. Plenum Press, New York, 1988.

[17] D. Dubois. Possibility theory and statistical reasoning. Computational Statistics and Data Analysis, 51(1):47-69, 2006.

[18] S. Ferson, V. Kreinovich, L. Ginzburg, D. S. Myers, and K. Sentz. Constructing probability boxes and Dempster-Shafer structures. Technical Report SAND2002-4015, Sandia National Laboratories, January 2003.

[19] S. Ferson and W. Tucker. Sensitivity analysis using probability bounding. Reliability Engineering and System Safety, 91(10-1):1435-1442, 2006.

[20] P. C. Fishburn. Interval Orders and Interval Graphs. Wiley, New York, 1985.

[21] M. Fuchs and A. Neumaier. Potential based clouds in robust design optimization. Journal of Statistical Theory and Practice, 3(1):225-238, 2008.

[22] I. Gilboa and D. Schmeidler. Maxmin expected utility with a non-unique prior. Journal of Mathematical Economics, 18:141-153, 1989.

[23] I. R. Goodman. Fuzzy sets as equivalence classes of random sets. In R. R. Yager, editor, Fuzzy Sets and Possibility Theory, pages 327-342. Pergamon Press, Oxford, 1982.

[24] M.J. Goovaerts, R. Kaas, A.E. Van Heerwaarden, and T. Bauwelinckx. Effective Actuarial Methods. North Holland, 1990.

[25] E. Hüllermeier. Cased-based approximate reasoning. Springer, 2007.

[26] M. Islam and J. Braden. Bio-economics development of floodplains: Farming versus fishing in bangladesh. Environment and Development Economics, 11(1):95-126, 2006.

[27] V. Krätschmer. When fuzzy measures are upper envelopes of probability measures. Fuzzy Sets and Systems, 138:455-468, 2003.

[28] E. Kriegler. Utilizing belief functions for the estimation of future climate change. International Journal of Approximate Reasoning, 39(2-3):185-209, 2005. 
[29] I. Levi. Potential surprise: its role in inference and decision making. In L. J. Cohen and M. Hesse, editors, Applications of Inductive Logic, pages 1-27. Clarendon Press, Oxford, 1980.

[30] H. Levy. Stochastic dominance. Kluwer Academic Publishers, 1998.

[31] M. Lorenz. Methods of measuring the concrentration of wealth. Publications of the American Statistical Association, 9(209-219):70, 1905.

[32] E. Miranda. A survey of the theory of coherent lower previsions. International Journal of Approximate Reasoning, 48(2):628-658, 2008.

[33] E. Miranda, I. Couso, and P. Gil. Upper probabilities attainable by distributions of measurable selections. Information Sciences, 180(8):107-1417, 2010.

[34] E. Miranda, G. de Cooman, and E. Quaeghebeur. Finitely additive extensions of distribution functions and moment sequences: The coherent lower prevision approach. International Journal of Approximate Reasoning, 48(1):132-155, 2008.

[35] E. Miranda, M. Troffaes, and S. Destercke. Generalised p-boxes on totally ordered spaces. In D. Dubois, M. Lubiano, H. Prade, M. Gil, P. Grzegiorzewski, and O. Hryniewicz, editors, Soft Methods for Handling Variability and Imprecision, pages 235-242. Springer, 2008.

[36] A. Müller and D. Stoyan. Comparison Methods for Stochastic Models and Risks. Wiley, 2002.

[37] N. Noyan, G. Rudolf, and A. Ruszczyński. Relaxations of linear programming problems with first order stochastic dominance constrains. Operations Research L, 34:653-659, 2006.

[38] M. Oberguggenberger and W. Fellin. Reliability bounds through random sets: non-parametric methods and geotechnical applications. Computers and Structures, 86(10):1093-1101, 2008.

[39] A. Owen. Nonparametric likelihood confidence bands for a distribution function. Journal of the American Statistical Association, 90:516-521, 1995.

[40] E. Raufaste, R. Da Silva Neves, and C. Mariné. Testing the descriptive validity of possibility theory in human judgements of uncertainty. Artificial Intelligence, 148:197-218, 2003.

[41] M. Rotschild and J.E. Stiglitiz. Increasing risk: a definition. Journal of Economic Theory, 2:225-243, 1970.

[42] J. K. Satia and R. E. Lave. Markovian decision processes with uncertain transition probabilities. Operations Research, 21:728-740, 1973.

[43] G. Shafer. A Mathematical Theory of Evidence. Princeton University Press, Princeton, NJ, 1976.

[44] M. Shaked and J. G. Shanthikumar. Stochastic Orders and their applications. Springer, 2006. 
[45] C. Strobl, A. L. Boulesteix and T. Augustin. Unbiased split selection for classification trees based on the Gini index. Computational Statistics and Data Analysis, 52(1), 483-501, 2007.

[46] H. Tanaka and P.J. Guo. Possibilistic Data Analysis for Operations Research. Physica-Verlag, Heidelberg, 1999

[47] M. Troffaes, E. Miranda, and S. Destercke. On the connection between probability boxes and possibility measures. In Proceedings of EUSFLAT'2011, pages 836-843, Aix-les-Bains, France, 2011.

[48] P. Walley. Statistical Reasoning with Imprecise Probabilities. Chapman and Hall, London, 1991.

[49] K. Weichselberger. Elementare Grundbegriffe einer allgemeineren Wahrscheinlichkeitsrechnung. I. Intervallwahrscheinlichkeit als umfassendes Konzept. Physica-Verlag Heidelberg, June 2001.

[50] M. Zaffalon, K. Wesnes, and O. Petrini. Reliable diagnoses of dementia by the naive credal classifier inferred from incomplete cognitive data. Artificial Intelligence in Medicine, 29:61-79, 2003.

\section{Appendix. Examples}

We have gathered in this section a number of counterexamples showing that the propositions established in this paper cannot be improved, in the sense that the implications we have not established do not hold in general. In the examples, we shall work for the most part with distribution functions associated to probability measures with finite support. Given $\vec{a}=\left(a_{1}, \ldots, a_{n}\right)$ such that $a_{1}+\ldots+a_{n}=1$, and $\vec{t}=\left(t_{1}, \ldots, t_{n}\right)$ with $t_{1} \leq \ldots \leq t_{n}$, the function $F_{\vec{a}, \vec{t}}$ corresponds to the cumulative distribution function of the probability measure $P_{\vec{a}, \vec{t}}$ satisfying $P_{\vec{a}, \vec{t}}\left(t_{i}\right)=a_{i}$ for $i=1, \ldots, n$. The only continuous distribution function we shall consider is the identity $F=i d$, defined by $F(x)=i d(x)=$ $x \forall x \in[0,1]$.

\section{Example 1. [The converse implications of Proposition 2 do not hold]}

By considering $\mathcal{F}_{1}=\left\{F_{1,0}, F_{1,1}\right\}, \mathcal{F}_{2}=\left\{F_{1,0.5}\right\}$, we can show that:

- $\succ_{F S D_{2}} \nRightarrow \succeq_{\mathrm{FSD}_{1}}, \succeq_{\mathrm{FSD}_{5}}, \succeq_{\mathrm{FSD}_{6}}$.

- $\succ_{F S D_{3}} \nRightarrow \succeq_{\mathrm{FSD}_{1}}, \succeq_{\mathrm{FSD}_{5}}, \succeq_{\mathrm{FSD}_{6}}$.

- $\succ_{F S D_{5}} \not \succeq_{\mathrm{FSD}_{1}}, \succeq_{\mathrm{FSD}_{2}}, \succeq_{\mathrm{FSD}_{3}}$.

- $\succ_{F S D_{6}} \nRightarrow \succeq_{\mathrm{FSD}_{1}}, \succeq_{\mathrm{FSD}_{2}}, \succeq_{\mathrm{FSD}_{3}}$.

- $\succeq_{\mathrm{FSD}_{4}} \nRightarrow \succeq_{\mathrm{FSD}_{\mathrm{i}}}$, for $i=1,2,3,5,6$.

To see that $\succeq_{F S D_{3}} \nRightarrow \succeq_{F S D_{2}}$ and $\succeq_{F S D_{6}} \nRightarrow \succeq_{F S D_{5}}$, take $\mathcal{F}_{1}=\mathcal{F}_{2}=\left\{F_{1, t} \mid\right.$ $t \in(0,1)\}$.

Let us turn now to strict stochastic dominance.

- With $\mathcal{F}_{1}=\left\{F_{1,1}\right\}, \mathcal{F}_{2}=\left\{F_{1,0}, F_{1,1}\right\}$, we can show that

$$
\succ_{F S D_{1}}, \succ_{F S D_{5}}, \succ_{F S D_{6}} \not \succ_{F S D_{2}}, \succ_{F S D_{3}}, \succ_{F S D_{4}} .
$$


- If we consider $\mathcal{F}_{1}=\left\{F_{1,0}, F_{1,1}\right\}, \mathcal{F}_{2}=\left\{F_{1,0}\right\}$, we deduce that

$$
\begin{aligned}
\succ_{F S D_{1}} & \nRightarrow \succ_{F S D_{5}}, \succ_{F S D_{6}} \\
\succ_{F S D_{2}}, \succ_{F S D_{3}} & \nRightarrow \succ_{F S D_{4}} .
\end{aligned}
$$

- If we take $\mathcal{F}_{1}=\left\{F, F_{(0.6,0.4),(0,1)}\right\}, \mathcal{F}_{2}=\left\{F_{1,0.5}, F_{(0.4,0.6),(0,1)}\right.$, we deduce that $\succ_{F S D_{4}} \nRightarrow \succ_{F S D_{1}}, \succ_{F S D_{2}}, \succ_{F S D_{3}}, \succ_{F S D_{5}}, \succ_{F S D_{6}}$.

- To see that $\succ_{F S D_{3}} \nRightarrow \succ_{F S D_{2}}$ and $\succ_{F S D_{6}} \nRightarrow_{F S D_{5}}$, consider the sets $\mathcal{F}_{1}=$ $\left\{F_{\left(\frac{1}{n}, 1-\frac{2}{n}, \frac{1}{n}\right),(0,0.5,1)} \mid n \geq 3\right\}$ and $\mathcal{F}_{2}=\mathcal{F}_{1} \cup\left\{F_{1,0.5}\right\}$.

Example 2. [The converse implications in Proposition 3 do not hold] Take $\mathcal{F}_{1}=\left\{F_{(0.3,0.7),(0,1)}, F_{(0.2,0.8),(0.2,0.3)}\right\}, \mathcal{F}_{2}=\{F\}$. They are incomparable under any of the definitions but $\underline{F}_{1} \leq \underline{F}_{2}=F=\bar{F}_{2} \leq \bar{F}_{1}$, from which we deduce that the converse implications in Proposition 3 do not hold.

Example 3. [Possible scenarios under Proposition 6]

- For $\equiv_{F S D_{i}}, i=1, \ldots, 6$, take $\mathcal{F}=\left\{I_{[0.5,1]}\right\}$.

- For $\mathcal{F} \succ_{F S D_{i}} \tilde{\mathcal{F}}$ for $i=1, \ldots, 6$, take $\mathcal{F}=\left\{I_{(0.5,1]}\right\}$.

- For $F S D_{i}$-incomparability, $i=1,2,5$, take $\mathcal{F}=\left\{I_{[x, 1]}: x \in(0,1)\right\}$.

\section{Example 4. [Possible scenarios under Proposition 7]}

- For $\mathcal{F}_{1} \succ_{F S D_{i}} \mathcal{F}_{2}, i=1,5,6$, take $\mathcal{F}_{1}=\{F\}$ and $\mathcal{F}_{2}=\left\{F, F_{1,0}\right\}$.

- For $\mathcal{F}_{2} \succ_{F S D_{i}} \mathcal{F}_{1}, i=1,2,3$, take $\mathcal{F}_{1}=\{F\}$ and $\mathcal{F}_{2}=\left\{F, F_{1,1}\right\}$.

- For $\mathcal{F}_{1} \equiv_{F S D_{i}} \mathcal{F}_{2}, i=1, \ldots, 6$, take $\mathcal{F}_{1}=\mathcal{F}_{2}=\{F\}$.

- Finally, if $\mathcal{F}_{1}=\mathcal{F}_{2}=\left\{F, F_{1,0.5}\right\}$ then $\mathcal{F}_{1}$ and $\mathcal{F}_{2}$ are $F S D_{i}$-incomparable for $i=1,2,5$.

\section{Example 5. [Possible scenarios under Proposition 8]}

- For $F S D_{i}$-incomparability, $i=1, \ldots, 6$, take $\mathcal{F}_{1}=\left\{F, F^{*}\right\}$, where $F^{*}=$ $\max \left\{F, F_{1,0.7}\right\}$, and $\mathcal{F}_{2}=\left\{F_{1,0.5}, F_{\{(0.5,0.5),(0,1)\}}\right\}$.

- With $\mathcal{F}_{1}=\left\{F, F^{*}\right\}$ and $\mathcal{F}_{2}=\left\{F_{(0.5,0.5),(0,0.5)}, F_{(0.5,0.5),(0.5,1)}\right\}$, we obtain $\mathcal{F}_{2} \succ_{F S D_{i}} \mathcal{F}_{1}$ for $i=2,3, \mathcal{F}_{1} \succ_{F S D_{i}} \mathcal{F}_{2}$ for $i=5,6$ and $\mathcal{F}_{1} \equiv_{F S D_{4}} \mathcal{F}_{2}$.

- With $\mathcal{F}_{1}=\left\{F, F^{*}\right\}, \mathcal{F}_{2}=\left\{F_{1,0.5}, F_{(0.5,0.5),(0,1)}, F_{(0.5,0.5),(0,0.5)}\right\}$, we get $\mathcal{F}_{1} \succ_{F S D_{4}} \mathcal{F}_{2}$. If instead $\mathcal{F}_{2}=\left\{F_{1,0.5}, F_{(0.5,0.5),(0,1)}, F_{(0.5,0.5),(0.5,1)}\right\}$, then $\mathcal{F}_{2} \succ_{F S D_{4}} \mathcal{F}_{1}$.

Example 6. [Possible scenarios under Proposition 10]

- For $F S D_{i}$-incomparability, $i=1, \ldots, 6$, take $\mathcal{F}_{1}=\left\{F_{\left(0.5-\frac{1}{n}, 0.5, \frac{1}{n}\right),(0,0.5,1)} \mid\right.$ $n \geq 3\}, \mathcal{F}_{2}=\left\{F_{1,0.5}, F_{(0.5,0.5),(0,1)}\right\}$.

- For $\mathcal{F}_{1} \equiv_{F S D_{i}} \mathcal{F}_{2}, i=4,5,6$, take $\mathcal{F}_{1}=\left\{F_{1,0.5}, F_{(0.5,0.5),(0,0.5)}\right\}, \mathcal{F}_{2}=$ $\left\{F_{(0.5,0.5),(0,0.5)}, F_{1,1}\right\}$.

- For $\mathcal{F}_{1} \succ_{F S D_{5}, F S D_{6}} \mathcal{F}_{2}, \mathcal{F}_{1}=\left\{F_{\left(1-\frac{1}{n}, \frac{1}{n}\right),(0,1)}: n \geq 3\right\}, \mathcal{F}_{2}=\left\{F_{1,0}, F_{1,1}\right\}$. 
- For $\mathcal{F}_{2} \succ_{F S D_{5}, F S D_{6}} \mathcal{F}_{1}$, consider the sets $\mathcal{F}_{1}=\left\{F_{1,0}, F_{(0.75,0.25),(0,1)}\right\}$ and $\mathcal{F}_{2}=\left\{F_{\left(1-\frac{1}{n}, \frac{1}{n}\right),(0,1)}: n \geq 3\right\}$

- For $\mathcal{F}_{1} \succ_{F S D_{4}} \mathcal{F}_{2}$, take $\mathcal{F}_{1}=\left\{F_{(0.6,0.4),(0.5,1)}, F_{\left(0.5-\frac{1}{n}, 0.5, \frac{1}{n}\right),(0,0.5,1)}: n \geq\right.$ $3\}, \mathcal{F}_{2}=\left\{F_{1,0.5}, F_{(0.5,0.5),(0,1)}\right\}$.

- Finally, for $\mathcal{F}_{2} \succ_{F S D_{2}, F S D_{3}, F S D_{4}} \mathcal{F}_{1}$, take $\mathcal{F}_{1}=\left\{F_{\left(0.5-\frac{1}{n}, 0.5, \frac{1}{n}\right),(0,0.5,1)}\right.$ : $n \geq 3\}, \mathcal{F}_{2}=\left\{F_{1,0.5}, F_{(0.5,0.5),(0,1)}, F_{(0.5,0.5),(0.5,1)}\right\}$.

\section{Example 7. [Possible scenarios under Proposition 11]}

- For $\mathcal{F}_{1} \succ_{F S D_{i}} \mathcal{F}_{2}, i=2,3$ and $\mathcal{F}_{2} \succ_{F S D_{i}} \mathcal{F}_{1}, i=5,6$, take

$$
\mathcal{F}_{1}=\left\{F_{(0.5,0.5),(0,0.5)}, F_{(0.5,0.5),(0.5,1)}\right\}, \mathcal{F}_{2}=\left\{F_{1,0.5}, F_{(0.5,0.5),(0,1)}\right\} .
$$

Reverse the roles of roles of $\mathcal{F}_{1}, \mathcal{F}_{2}$ to obtain $\mathcal{F}_{1} \succ_{F S D_{i}} \mathcal{F}_{2}$ for $i=5,6$ and $\mathcal{F}_{2} \succ_{F S D_{i}} \mathcal{F}_{1}$ for $i=2,3$.

- For $F S D_{i}$-incomparability, $i=1, \ldots, 6$, take $\mathcal{F}_{1}=\left\{F_{1,0.5}, F_{(0.5,0.5),(0,1)}\right\}$ and $\mathcal{F}_{2}=\left\{F_{\left(\frac{1}{n}, 0.5,0.5-\frac{1}{n}\right),(0,0.5,1)}, F_{\left(0.5-\frac{1}{n}, 0.5, \frac{1}{n}\right),(0,0.5,1)}: n \geq 3\right\}$.

- For $\mathcal{F}_{2} \succ_{F S D_{4}} \mathcal{F}_{1}$, take $\mathcal{F}_{1}=\left\{F_{1,0.5}, F_{(0.5,0.5),(0,1)}\right\}$ and $\mathcal{F}_{2}=\left\{F_{\left(\frac{1}{n}, 0.5,0.5-\frac{1}{n}\right),(0,0.5,1)}, F_{\left(0.5-\frac{1}{n}, 0.5, \frac{1}{n}\right),(0,0.5,1)}: n \geq 3, F_{(0.5,0.5),(0.5,1)}\right\}$. By reversing the roles of $\mathcal{F}_{1}, \mathcal{F}_{2}$ we obtain an example of $\mathcal{F}_{1} \succ_{F S D_{4}} \mathcal{F}_{2}$.

\section{Example 8. [Possible scenarios under Proposition 12]}

- For $\mathcal{F}_{1}^{*} \succ_{\mathrm{FSD}_{\mathrm{i}}} \mathcal{F}_{2}, i=2, \ldots, 6$, take $\mathcal{F}_{1}=\mathcal{F}_{1}^{*}$.

- To see that we may have $\mathcal{F}_{1} \succ_{\mathrm{FSD}_{3}} \mathcal{F}_{2}$ and $\mathcal{F}_{2} \succ_{\mathrm{FSD}_{3}} \mathcal{F}_{1}^{*}$, take the sets $\mathcal{F}_{1}=\left\{F_{(0.5,0.5),(0,0.5)}, F_{(0.5,0.5),(0.5,1)}\right\}, \mathcal{F}_{1}^{*}=\left\{F_{1,0.5}, F_{(0.5,0.5),(0,1)}\right\}$ and $\mathcal{F}_{2}=\left\{F_{(0.75,0.25),(0.5,1)}, F_{(0.25,0.25,0.5),(0,0.5,1)}\right\}$.

- For $\mathcal{F}_{1} \succ_{\mathrm{FSD}_{6}} \mathcal{F}_{2}$ and $\mathcal{F}_{2} \succ_{\mathrm{FSD}_{6}} \mathcal{F}_{1}^{*}$ take $\mathcal{F}_{1}, \mathcal{F}_{1}^{*}$ as before and $\mathcal{F}_{2}=$ $\left\{F_{(0.25,0.75),(0,0.5)}, F_{(0.5,0.25,0.25),(0,0.5,1)}\right\}$.

- For $\mathcal{F}_{1} \succ_{\mathrm{FSD}_{4}} \mathcal{F}_{2}$ and $\mathcal{F}_{2} \succ_{\mathrm{FSD}_{4}} \mathcal{F}_{1}^{*}$, let us consider

$$
\begin{aligned}
& \mathcal{F}_{1}=\left\{F_{(0.25,0.25,0.5),(0,0.5,1)}, F_{1,0.5}, F_{(0.5,0.5),(0,1)}\right\}, \\
& \mathcal{F}_{2}=\left\{F_{(0.25,0.5,0.25),(0,0.5,1)}, F_{(0.4,0.2,0.4),(0,0.5,1)}\right\}, \\
& \mathcal{F}_{1}^{*}=\left\{F_{\left.(0.25,0.75),(0,0.5), F_{1,0.5}, F_{(0.5,0.5),(0,1)}\right\} .}\right.
\end{aligned}
$$

- For $\mathcal{F}_{1}^{*} \equiv_{F S D_{i}} \mathcal{F}_{2}$ and $\mathcal{F}_{1} \succ_{F S D_{i}} \mathcal{F}_{2}, i=3,4,6$, consider $\mathcal{F}_{1}, \mathcal{F}_{2}$ associated to the same $p$-box and such that $\mathcal{F}_{1} \succ_{F S D_{i}} \mathcal{F}_{2}$ for $i=3, \ldots, 6$, as in Example 7 , and let $\mathcal{F}_{1}^{*}=\mathcal{F}_{2}$.

- For $\mathcal{F}_{1}^{*} \equiv_{F S D_{5}} \mathcal{F}_{2}$ and $\mathcal{F}_{1} \succ_{F S D_{5}} \mathcal{F}_{2}$, let $\mathcal{F}_{1}=\left\{F_{1,0.5}, F_{(0.5,0.5),(0,1)}\right\}, \mathcal{F}_{2}=$ $\left\{F_{(0.5,0.5),(0,0.5)}, F_{(0.5,0.5),(0.5,1)}\right\}$ and $\mathcal{F}_{1}^{*}=\mathcal{F}_{2}$.

- For $\mathcal{F}_{1} \succ_{F S D_{i}} \mathcal{F}_{2}$ while $\mathcal{F}_{1}^{*}, \mathcal{F}_{2}$ are $F S D_{i}$-incomparable for $i=2,3,4$, take $\mathcal{F}_{1}=\left\{F_{(0.5,0.5),(0.5,1)}, F_{(0.5,0.5),(0,0.5)}\right\}, \mathcal{F}_{2}=\{F\}$ and let us consider $\mathcal{F}_{1}^{*}=\left\{F_{1,0.5}, F_{(0.5,0.5),(0,1)}\right\}$. 
- For $\mathcal{F}_{1} \succ_{F S D_{6}} \mathcal{F}_{2}$ while $\mathcal{F}_{1}^{*}, \mathcal{F}_{2}$ are $F S D_{6}$-incomparable, take

$$
\begin{aligned}
& \mathcal{F}_{1}=\left\{F_{\left(\frac{1}{n}, 1-\frac{2}{n}, \frac{1}{n}\right),(0,0.5,1)}, F_{\left(\frac{1}{2}-\frac{1}{n}, \frac{2}{n}, \frac{1}{2}-\frac{1}{n}\right),(0,0.5,1)} \mid n \geq 3\right\}, \\
& \mathcal{F}_{1}^{*}=\left\{F_{1,0.5}, F_{(0.5,0.5),(0,1)}\right\}, \\
& \mathcal{F}_{2}=\left\{F_{\left(0.5-\frac{1}{n}, 0.5, \frac{1}{n}\right),(0,0.5,1)}, F \mid n \geq 3\right\} .
\end{aligned}
$$

Example 9. [The converse implications of Proposition 17 do not hold.]

- Given $\mathcal{F}_{1}=\left\{F_{1,0.5-\frac{1}{n}}: n>3\right\}, \mathcal{F}_{2}=\left\{F_{1,0.5}\right\}$, we have that $x_{\underline{F}_{1}}=x_{\underline{F}_{2}}=$ $x_{\bar{F}_{2}}=0.5$, but $\mathcal{F}_{1} \nsucceq_{F S D_{2}, F S D_{3}, F S D_{4}} \mathcal{F}_{2}$.

- Given $\mathcal{F}_{1}=\left\{F_{1,0.5+\frac{1}{n}}: n>3\right\}, \mathcal{F}_{2}=\left\{F_{1,0.5}\right\}$, we have $x_{\bar{F}_{1}}=x_{\bar{F}_{2}}=0.5$, but $\mathcal{F}_{1} \nsucceq_{F S D_{5}, F S D_{6}} \stackrel{\mathcal{F}_{2}}{\mathcal{F}_{2}}$.

- For the second item, take $\mathcal{F}_{1}=\mathcal{F}_{2}=\left\{I_{[0.5,1]}\right\}$; then $\mathcal{F}_{1} \equiv_{F S D_{i}} \mathcal{F}_{2}$ for $i=1, \ldots, 6$, but no strict inequality holds. 